\title{
A bi-dissipative damage model for concrete
}

\section{Um modelo de dano bi-dissipativo para o concreto}
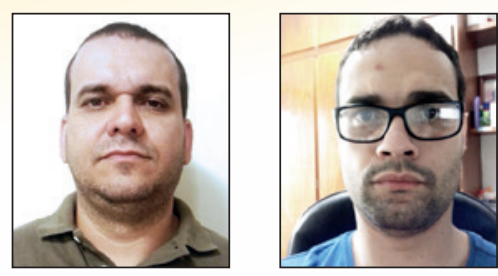

J. J. C. PITUBA a Julio_pituba@ufg.br

W. M. PEREIRA JÚNIOR b w.junioreng@gmail.com

\begin{abstract}
This work deals with an improvement of an anisotropic damage model in order to analyze reinforced concrete structures submitted to reversal loading. The original constitutive model is based on the fundamental hypothesis of energy equivalence between real and continuous media following the concepts of the Continuum Damage Mechanics. The concrete is assumed as an initial elastic isotropic medium presenting anisotropy, permanent strains and bimodularity induced by damage evolution. In order to take into account the bimodularity, two damage tensors governing the rigidity in tension or compression regimes are introduced. However, the original model is not capable to simulate the influence of the previous damage processes in compression regimes. In order to avoid this problem, some conditions are introduced to simulate the damage unilateral effect. It has noted that the damage model is agreement with to micromechanical theory conditions when dealing to unilateral effect in concrete material. Finally, the proposed model is applied in the analyses of reinforced concrete framed structures submitted to reversal loading. These numerical applications show the good performance of the model and its potentialities to simulate practical problems in structural engineering.
\end{abstract}

Keywords: damage mechanics, unilateral effect, concrete structures, structural failure.

\section{Resumo}

Este trabalho trata do aperfeiçoamento de um modelo dano anisótropo de modo a analisar estruturas em concreto armado submetidas à inversão de carregamento. O modelo constitutivo original é baseado nas hipóteses fundamentais de equivalência de energia entre meio real danificado e meio contínuo equivalente segundo os conceitos da Mecânica do Dano no Contínuo. O concreto é assumido como um meio inicialmente elástico e isótropo que passa a apresentar comportamento mecânico anisótropo, plástico e bimodular induzidos pelos processos de danificação. Com o intuito de se levar em conta a bimodularidade do meio, são propostos dois tensores de dano governando a rigidez em regimes de tração e de compressão. Entretanto, o modelo original não é capaz de simular a influência de processos prévios de danificação em regimes de compressão. Objetivando contornar este problema, algumas condições são introduzidas no modelo para simular o efeito unilateral causado pelo dano. Observa-se que o modelo de dano está de acordo com as condições obtidas da Teoria Micromecânica quando tratando do efeito unilateral em materiais frágeis, como o concreto. Finalmente, o modelo de dano proposto é aplicado na análise de estruturas de pórticos de concreto armado submetidas a cargas com inversão de sinal. Os resultados comprovam o bom desempenho do modelo e potencialidades para simular problemas práticos da Engenharia Estrutural.

Palavras-chave: mecânica do dano, efeito unilateral, estruturas de concreto, falha estrutural. 


\section{Introduction}

The Continuum Damage Mechanics (CDM) has already proved to be a suitable tool for simulating the material deterioration in equivalent continuous media due exclusively to microcracking process. In this work, for modeling the concrete behavior, it can be assumed that the concrete belongs to the category of materials which can be considered initially isotropic and unimodular presenting different behaviors in tension and compression when damaged. A formulation of constitutive laws for isotropic and anisotropic elastic materials presenting different behaviors in tension and compression under small deformations was proposed in Curnier [1] for two and three-dimensional cases. The authors have considered a bimodular hyperelastic material defining an elastic potential energy density $W$ which must be once continuously differentiable (whole wise), but only piecewise twice continuously differentiable. In this way, the mode is able to produce different response in tension and compression. Pituba [2] has extended that formulation in order to take into account the damage effects. Accordingly with, the bulk $\left(\lambda_{a b}\right)$ and shear $\left(\mu_{a}\right)$ moduli are considered as functions of the damage state, so that the stress-strain relationship would be influenced by damage variables. Moreover, the hypersurface $g\left(\varepsilon, D_{i}\right)$ adopted as the criterion for identification of the constitutive responses in compression or tension would be also influenced by the damage variables. Then, a damage constitutive model accounting for induced anisotropy and bimodular elastic response for the concrete was derived from Pituba [2] and its potentialities for $1 \mathrm{D}$ and $2 \mathrm{D}$ analyses are discussed in Pituba [3], Pituba and Fernandes [4], Pituba and Lacerda [5] and Pituba [6]. Besides, the simulations of experimental tests in uniaxial, biaxial and triaxial stress tests are reported in Pituba and Fernandes [4]. The original version of the damage mode is bimodular in the sense that presents different elasticity tensors in tension and compression. Thus, the model is potentially capable to simulate the stiffness recovery when the medium is submitted to a reversal loading that evidences the transition from predominant regimes of tension to compression, i. e., the so-called unilateral behavior of the damaged concrete. However, the model is not capable to simulate the influence of the previous damage processes in compression (diffuse damage) when there is the transition from predominant regimes of compression to tension Comi [7]. From a micromechanics point of view this characteristic is due to the partial closure of micro-cracks loaded in compression which affect less the elasticity moduli in compression than in tension, Desmorat [8] Therefore, to avoid this problem a new elasticity tensor is proposed and some numerical analyses are performed to simulate practical problems in structural engineering.

Many different strategies are possible and have been proposed in the literature to model the stiffness recovery as described in Comi [7], Carol and Willam [9], Welemane and Comery [10], Bielski et al. [11], Liu [12] and Araújo and Proença [13]. For more details, in Bielski [11] is presented a summary of some formulations of models that take into account the unilateral effect of the damage process, such as: the use of fourth-rank projection operators for the decomposition of the stress and strain tensors into the positive and negative projections, besides the use of the generalized projection operators.
On the other hand, despite the progresses in the macroscopic modeling of the unilateral effect (in particular, the continuity problems that arise when the induced anisotropy is simultaneously described), this subject still remains as an open research field when it deals with induced anisotropy damage models, even when the micromechanical theory has been used to justify the proposal of constitutive models dealing with cracked media, Welemane and Comery [10], Zhu [14], Zhu [15] and Pichler and Dormieux [16]. This can be noted even when dealing with more actual approaches based on multi-scale analysis procedures, Skarzynski and Tejchman [17] and Pituba and Souza Neto [18]. Indeed, this work intends to contribute to the modeling of damage unilateral effect applied to concrete structures. However, it must be noted that the proposed model is not capable to take into account the friction effects, namely blocking and dissipative sliding of closed microcrack lips. This feature can be discussed in future works.

\section{Bi-dissipative plastic-damage model}

\subsection{Original proposal of Pituba and Fernandes [4]}

The original damage model formulation Pituba and Fernandes [4] is built from the formalism presented in Pituba [2]. Moreover, the model respects the principle of energy equivalence between damaged real medium and equivalent continuous medium established in the CDM. The damage model is briefly presented in this work. Initially, for dominant tension states, a damage tensor is proposed:

$$
\begin{aligned}
& \boldsymbol{D}_{\boldsymbol{T}}=f_{1}\left(D_{1}, D_{4}, D_{5}\right)(\boldsymbol{A} \otimes \boldsymbol{A})+ \\
& 2 f_{2}\left(D_{4}, D_{5}\right)[(\boldsymbol{A} \bar{\otimes} \boldsymbol{I}+\boldsymbol{I} \otimes \boldsymbol{A})-(\boldsymbol{A} \otimes \boldsymbol{A})]
\end{aligned}
$$

where $f_{1}\left(D_{1}, D_{4}, D_{5}\right)=D_{1}-2 f_{2}\left(D_{4}, D_{5}\right)$ and $f_{2}\left(D_{4}, D_{5}\right)=1-\left(1-D_{4}\right)$ $\left(1-D_{5}\right)$. The variable $D_{1}$ represents the damage in direction orthogonal to the transverse isotropy local plane of the material, while $D_{4}$ is representative of the damage due to the sliding movement between the crack faces. The third damage variable, $D_{5}$, is only activated if a previous compression state accompanied by damage has occurred. In Curnier [1], the tensor I is the second-order identity tensor and the tensor $\mathbf{A}$, is formed by dyadic product of the unit vector perpendicular to the transverse isotropy plane for himself. Those products are given in Pituba [2]. For dominant compression states, it is proposed the other damage tensor:

$$
\begin{aligned}
& \boldsymbol{D}_{\boldsymbol{C}}=f_{1}^{*}\left(D_{2}, D_{4}, D_{5}\right)(\boldsymbol{A} \otimes \boldsymbol{A})+f_{2}\left(D_{3}\right)[(\boldsymbol{I} \otimes \boldsymbol{I})- \\
& (\boldsymbol{A} \otimes \boldsymbol{A})]+2 f_{3}\left(D_{4}, D_{5}\right) \\
& {[(\boldsymbol{A} \otimes \boldsymbol{I}+\boldsymbol{I} \otimes \boldsymbol{A})-(\boldsymbol{A} \otimes \boldsymbol{A})]}
\end{aligned}
$$

where $f_{1}^{*}\left(D_{2}, D_{4}, D_{5}\right)=D_{2}-2 f_{3}\left(D_{4}, D_{5}\right), f_{2}\left(D_{3}\right)=D_{3}$ and $f_{3}\left(D_{4}, D_{5}\right)=1-$ $\left(1-D_{4}\right)\left(1-D_{5}\right)$. Note that the compression damage tensor introduces two additional scalar variables in its composition: $D_{2}$ and $D_{3}$. The variable $D_{2}$ (damage perpendicular to the transverse isotropy local plane) reduces the Young's modulus in that direction and in conjunction to $D_{3}$ (that represents the damage in the transverse isotropy plane) degrades the Poisson's ratio throughout the perpendicular planes to the one of transverse isotropy. 
On the other hand, the constitutive tensor is written as:

$$
\mathbf{E}(\varepsilon):=\left\{\begin{array}{l}
E_{-}(\varepsilon) \text { if } g\left(\varepsilon, D_{T}, D_{C}\right)<0, \\
E_{+}(\varepsilon) \text { if } g\left(\varepsilon, D_{T}, D_{C}\right)>0,
\end{array}\right.
$$

$$
\begin{aligned}
& \boldsymbol{E}_{+}(\varepsilon)=\lambda_{11}[I \otimes I]+2 \mu_{1}[\bar{I}-\overline{-} I]- \\
& \lambda_{22}^{+}\left(D_{1}, D_{4}, D_{5}\right)[A \otimes A]-\lambda_{I 2}^{+}\left(D_{1}\right)[\mathbf{A} \otimes \mathbf{I}+\mathbf{I} \otimes \mathbf{A}] \\
& -\mu_{2}\left(D_{4}, D_{5}\right)[\bar{A} \otimes \boldsymbol{I}+\boldsymbol{I} \otimes \boldsymbol{A}]
\end{aligned}
$$

$$
H(D i)=1 \text { to } D i>0 ; H(D i)=0 \text { to } D i=0 \quad(i=1,2)
$$

where $\gamma_{1}\left(D_{1}, D_{2}\right)=\left\{1+H\left(D_{2}\right)\left[H\left(D_{1}\right)-1\right]\right\} \eta\left(D_{1}\right)+\left\{1+H\left(D_{1}\right)\left[H\left(D_{2}\right)-1\right]\right\}$ $\eta\left(D_{2}\right)$ and $\gamma_{2}\left(D_{1}, D_{2}\right)=D_{1}+D_{2}$.

The Heaveside functions employed above are given by:

The $\eta\left(D_{1}\right)$ and $\eta\left(D_{2}\right)$ functions are defined, respectively, for the tension and compression cases, assuming for the first one that there was no previous damage in compression affecting the present tension damage variable $D_{1}$ and analogously, for the second one that has not had previous tension damage affecting variable $D_{2}$. The proposed functions are:

$$
\eta\left(D_{1}\right)=\frac{-D_{1}+\sqrt{3-2 D_{1}^{2}}}{3}
$$

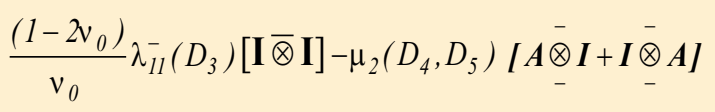

The remaining parameters will only exist for no-null damage, evidencing the anisotropy and bimodularity induced by damage. Those parameters are given by:

$$
\begin{aligned}
& \lambda_{22}^{+}\left(D_{1}, D_{4}, D_{5}\right)=\left(\lambda_{0}+2 \mu_{0}\right)\left(2 D_{1}-D_{1}^{2}\right)-2 \lambda_{12}^{+}\left(D_{1}\right)-2 \mu_{2}\left(D_{4}, D_{5}\right) \\
& \lambda_{12}^{+}\left(D_{1}\right)=\lambda_{0} D_{1} ; \mu_{2}\left(D_{4}, D_{5}\right)=2 \mu_{0}\left[1-\left(1-D_{4}\right)^{2}\left(1-D_{5}\right)^{2}\right] \\
& \lambda_{22}^{-}\left(D_{2}, D_{3}, D_{4}, D_{5}\right)=\left(\lambda_{0}+2 \mu_{0}\right)\left(2 D_{2}-D_{2}^{2}\right)-2 \lambda_{12}^{2}\left(D_{2}, D_{3}\right)+\frac{\left(v_{0}-1\right)}{v_{1}} \lambda_{11}^{-}\left(D_{3}\right)-2 \mu_{2}\left(D_{4}, D_{5}\right) \\
& \lambda_{12}^{-}\left(D_{2}, D_{3}\right)=\lambda_{0}\left[\left(1-D_{3}\right)^{2}-\left(1-D_{2}\right)\left(1-D_{3}\right)\right] ; \lambda_{11}^{-}\left(D_{3}\right)=\lambda_{0}\left(2 D_{3}-D_{3}^{2}\right)
\end{aligned}
$$

$$
\eta\left(D_{2}\right)=\frac{-D_{2}+\sqrt{3-2 D_{2}^{2}}}{3}
$$

Note that if the damage process in the material is not activated $\left(D_{1}=\right.$ $D_{2}=0$ ) the Eq. (8) recovers the equation proposed by Comi [7], thus the formulation satisfies the proposed condition of initially isotropic material. Already, if the material is totally damaged, $D_{1}=D_{2}=1\left(\eta\left(D_{1}\right)\right.$ $\left.=\eta\left(D_{2}\right)=0\right)$ and $\gamma_{2}=2$, the hyperplane $g(\varepsilon)$ is coincident to the transverse isotropy local plane of the material and, therefore, the normal vector to the hyperplane is given by the transverse isotropy tensor $\mathbf{A}$. On the other hand, due to anisotropy induced by damage, it is convenient to separate the damage criterion into two criteria: the first one is only used to indicate the beginning of the damage processes, or that the material is no longer isotropic; the second one is used for loading and unloading processes, when the material is already considered as transverse isotropic medium. This second criterion identifies if there is or not evolution of the damage variables. That division is justified by the difference between the complementary elastic strain energies of isotropic and transverse isotropic materials. If there is damage evolution, i. e., when $\dot{\mathbf{D}}_{\mathrm{T}} \neq \mathbf{0}$ or $\dot{\mathbf{D}}_{\mathbf{C}} \neq \mathbf{0}$, the evolution laws of the damage variables are written as associated variables functions. Considering just the case of monotonic loading, the evolution laws proposed for the scalar damage variables are resulting of fittings on experimental results. The general form proposed is:

$$
D_{i}=1-\frac{1+A_{i}}{A_{i}+\exp \left[B_{i}\left(Y_{i}-Y_{0 i}\right)\right]} i=1,5
$$
strain and damage states. Accordingly with Eq. (7) and referring to general cases of loading, the following relationship has been proposed for the hyperplane: 
where $A_{i}, B_{i}$ and $Y_{O i}$ are parameters of the model that must be identified through the uniaxial tension and compression tests and biaxial compression tests.

When the damage process is activated, the formulation starts to involve the tensor $\mathbf{A}$ that depends on the normal to the transverse isotropy plane. Therefore, it is necessary to establish some rules to identify its location for an actual strain state. Initially, it is established a general criterion for the existence of the transverse isotropy plane. In Pituba and Fernandes [4] is proposed that the transverse isotropy due to damage only arises if positive strain rates exist at least in one of the principal directions. After assuming such proposition as valid, some rules to identify its location are defined.

\subsection{Discussion about the unilateral effect in brittle materials}

The original version of the damage model is bimodular, however it is necessary to take into account the diffuse damage generated in previous compression regimes when dealing with tension regimes. This problem can be solved by introduction of a new elasticity tensor in tension dominant states. Therefore, respecting the principle of energy equivalence, for the uniaxial point of view, for instance, the constitutive tensor is written as:

$$
\boldsymbol{E}_{\boldsymbol{T}}=\boldsymbol{E}_{0}\left(1-D_{1}\right)^{2}\left(1-D_{2}\right)^{2}
$$

The relationship above shows that in tension dominant states prevailing prior to activation of damage in compression is possible to solve the problem discussed here. By analogy, under multiaxial stress states, it can be concluded that damage tensor in compression $\mathbf{D}_{\mathrm{c}}$ should compose the expression of the constitutive tensor in tension dominant states. Therefore, respecting the principle of equivalence of energy, the constitutive tensor is now written as:

$$
\mathbf{E}_{\mathbf{T}}=\left(\mathbf{I}-\mathbf{D}_{\mathbf{C}}\right)\left(\mathbf{I}-\mathbf{D}_{\mathrm{T}}\right) \mathbf{E}_{\mathbf{0}}\left(\mathbf{I}-\mathbf{D}_{\mathrm{T}}\right)\left(\mathbf{I}-\mathbf{D}_{\mathbf{C}}\right)
$$

Considering a matrix representation and assuming, for instance, that the transversal isotropy local plane is coincident to the 2-3 plane, the constitutive tensor $\mathbf{E}_{\mathrm{T}}$ may be described as follows:

$$
\boldsymbol{E}_{T}=\left[\begin{array}{cccccc}
\left(\lambda_{0}+2 \mu_{0}\right)\left(1-\boldsymbol{D}_{1}\right)^{2}\left(1-\boldsymbol{D}_{2}\right)^{2} & \lambda_{0}\left(1-\boldsymbol{D}_{1}\right)\left(1-\boldsymbol{D}_{2}\right)\left(1-\boldsymbol{D}_{3}\right) & \lambda_{0}\left(1-\boldsymbol{D}_{1}\right)\left(1-\boldsymbol{D}_{2}\right)\left(1-\boldsymbol{D}_{3}\right) & 0 & 0 & 0 \\
\lambda_{0}\left(1-\boldsymbol{D}_{1}\right)\left(1-\boldsymbol{D}_{2}\right)\left(1-\boldsymbol{D}_{3}\right) & \left(\lambda_{0}+2 \mu_{0}\right)\left(1-\boldsymbol{D}_{3}\right)^{2} & \lambda_{0}\left(1-\boldsymbol{D}_{3}\right)^{2} & 0 & 0 & 0 \\
\lambda_{0}\left(1-\boldsymbol{D}_{1}\right)\left(1-\boldsymbol{D}_{2}\right)\left(1-\boldsymbol{D}_{3}\right) & \lambda_{0}\left(1-\boldsymbol{D}_{3}\right)^{2} & \left(\lambda_{0}+2 \mu_{0}\right)\left(1-\boldsymbol{D}_{3}\right)^{2} & 0 & 0 & 0 \\
0 & 0 & 0 & 2 \mu_{0} & 0 & 0 \\
0 & 0 & 0 & 0 & 2 \mu_{0}\left(1-\boldsymbol{D}_{4}\right)^{4}\left(1-\boldsymbol{D}_{5}\right)^{4} & 0 \\
0 & 0 & 0 & 0 & 0 & 2 \mu_{0}\left(1-\boldsymbol{D}_{4}\right)^{4}\left(1-\boldsymbol{D}_{5}\right)^{4}
\end{array}\right]
$$

It can be noted that the equations (5) and (11) present different values for the shear moduli in compression and tension dominant states, respectively. Therefore, this alternative formulation in order to take into account the diffuse damage does not respect the Curnier's condition about the tangential continuity. To avoid this problem, another expression for the damage tensor in compression dominant states $\mathbf{D}_{\mathbf{C}}^{*}$ is proposed. This tensor is given by:

$$
\boldsymbol{D}_{C}^{*}=f_{1}\left(D_{2},\right)(\boldsymbol{A} \otimes \boldsymbol{A})+f_{2}\left(D_{3}\right)[(\boldsymbol{I} \otimes \boldsymbol{I})-(\boldsymbol{A} \otimes \boldsymbol{A})]
$$

where $f_{1}\left(D_{2}\right)=D_{2}$ and $f_{2}\left(D_{3}\right)=D_{3}$. It is important to observe that the damage tensor $\mathbf{D}_{\mathbf{C}}^{*}$ provides the diffuse damage in previous compression states through the changing of the volumetric modulus, as proposed in Comi [7]. For simplicity, considering a matrix representation and assuming, for instance, that the trans- versal isotropy local plane is coincident to the $2-3$ plane, equation (16) is written as:

$\boldsymbol{D}_{\boldsymbol{C}}^{*}=\left[\begin{array}{cccccc}\boldsymbol{D}_{2} & 0 & 0 & 0 & 0 & 0 \\ 0 & \boldsymbol{D}_{3} & 0 & 0 & 0 & 0 \\ 0 & 0 & \boldsymbol{D}_{3} & 0 & 0 & 0 \\ 0 & 0 & 0 & 0 & 0 & 0 \\ 0 & 0 & 0 & 0 & 0 & 0 \\ 0 & 0 & 0 & 0 & 0 & 0\end{array}\right]$

Finally, taking into account the principle of energy equivalence, the constitutive tensor for tension dominant states is given by:

$$
\mathbf{E}_{\mathrm{T}}=\left(\mathbf{I}-\mathbf{D}_{\mathrm{C}}^{*}\right)\left(\mathbf{I}-\mathbf{D}_{\mathrm{T}}\right) \mathbf{E}_{0}\left(\mathbf{I}-\mathbf{D}_{\mathrm{T}}\right)\left(\mathbf{I}-\mathbf{D}_{\mathrm{C}}^{*}\right)
$$




$$
\boldsymbol{E}_{T}=\left[\begin{array}{cccccc}
\left(\lambda_{0}+2 \mu_{0}\right)\left(1-\boldsymbol{D}_{1}\right)^{2}\left(1-\boldsymbol{D}_{2}\right)^{2} & \lambda_{0}\left(1-\boldsymbol{D}_{1}\right)\left(1-\boldsymbol{D}_{2}\right)\left(1-\boldsymbol{D}_{3}\right) & \lambda_{0}\left(1-\boldsymbol{D}_{1}\right)\left(1-\boldsymbol{D}_{2}\right)\left(1-\boldsymbol{D}_{3}\right) & 0 & 0 & 0 \\
\lambda_{0}\left(1-\boldsymbol{D}_{1}\right)\left(1-\boldsymbol{D}_{2}\right)\left(1-\boldsymbol{D}_{3}\right) & \left(\lambda_{0}+2 \mu_{0}\right)\left(1-\boldsymbol{D}_{3}\right)^{2} & \lambda_{0}\left(1-\boldsymbol{D}_{3}\right)^{2} & 0 & 0 & 0 \\
\lambda_{0}\left(1-\boldsymbol{D}_{1}\right)\left(1-\boldsymbol{D}_{2}\right)\left(1-\boldsymbol{D}_{3}\right) & \lambda_{0}\left(1-\boldsymbol{D}_{3}\right)^{2} & \left(\left(\lambda_{0}+2 \mu_{0}\right)\left(1-\boldsymbol{D}_{3}\right)^{2}\right. & 0 & 0 & 0 \\
0 & 0 & 0 & 2 \mu_{0} & 0 & 0 \\
0 & 0 & 0 & 0 & 2 \mu_{0}\left(1-\boldsymbol{D}_{4}\right)^{2}\left(1-\boldsymbol{D}_{5}\right)^{2} & 0 \\
0 & 0 & 0 & 0 & 0 & 2 \mu_{0}\left(1-\boldsymbol{D}_{4}\right)^{2}\left(1-\boldsymbol{D}_{5}\right)^{2}
\end{array}\right]
$$

Then, following the formalism presented in Pituba [2], the bidissipative anisotropy damage model taking into account the unilateral effect in brittle materials is written as:

$$
\mathrm{W}(\varepsilon)=\rho \psi(\varepsilon):=\left\{\begin{array}{l}
W_{-}(\varepsilon) \text { if } \boldsymbol{g}\left(\varepsilon, \boldsymbol{D}_{\boldsymbol{T}}, \boldsymbol{D}_{\boldsymbol{C}}\right)<0 \\
W_{+}(\varepsilon) \text { if } \boldsymbol{g}\left(\varepsilon, \boldsymbol{D}_{\boldsymbol{T}}, \boldsymbol{D}_{\boldsymbol{C}}\right)>0
\end{array}\right.
$$

$$
\begin{aligned}
& \mathrm{W}_{+}=\rho \psi_{+}(\varepsilon)=\frac{\lambda_{11}}{2} \operatorname{tr}^{2}(\varepsilon)+\mu_{1} \operatorname{tr}\left(\varepsilon^{2}\right)-\frac{\lambda_{22}^{+}\left(D_{1}, D_{2}, D_{3}, D_{4}, D_{5}\right)}{2} \operatorname{tr}^{2}(\mathbf{A} \varepsilon)-\lambda_{12}^{+}\left(\mathrm{D}_{1}, \mathrm{D}_{2}, \mathrm{D}_{3}\right) \operatorname{tr}(\varepsilon) \operatorname{tr}(\mathbf{A} \varepsilon) \\
& -\frac{\lambda_{11}^{-}\left(D_{3}\right)}{2} \operatorname{tr}^{2}(\varepsilon)-\frac{\left(1-2 v_{0}\right)}{2 v_{0}} \lambda_{11}^{-}\left(D_{3}\right) \operatorname{tr}[(\boldsymbol{I} \bar{\otimes} \boldsymbol{I}) \varepsilon]^{2}-\mu_{2}\left(D_{4}, D_{5}\right) \operatorname{tr}\left(A \varepsilon^{2}\right)
\end{aligned}
$$

$\mathrm{W}_{-}=\rho \psi_{-}(\varepsilon)=\frac{\lambda_{11}}{2} \operatorname{tr}^{2}(\varepsilon)+\mu_{1} \operatorname{tr}\left(\varepsilon^{2}\right)-\frac{\lambda_{22}^{-}\left(D_{2}, D_{3}, D_{4}, D_{5}\right)}{2} \operatorname{tr}^{2}(\mathbf{A} \varepsilon)-\lambda_{12}^{-}\left(\mathrm{D}_{2}, \mathrm{D}_{3}\right) \operatorname{tr}(\varepsilon) \operatorname{tr}(\mathbf{A} \varepsilon)$

$$
-\frac{\lambda_{11}^{-}\left(D_{3}\right)}{2} \operatorname{tr}^{2}(\varepsilon)-\frac{\left(1-2 v_{0}\right)}{2 v_{0}} \lambda_{11}^{-}\left(D_{3}\right) \operatorname{tr}[(\boldsymbol{I} \bar{\otimes} \boldsymbol{I}) \varepsilon]^{2}-\mu_{2}\left(D_{4}, D_{5}\right) \operatorname{tr}\left(\boldsymbol{A} \varepsilon^{2}\right)
$$

Now, the parameters $\lambda_{\mathrm{ij}}$ and $\mu_{\mathrm{i}}$ are given by:

$$
\begin{aligned}
& \lambda_{22}^{+}\left(D_{1}, D_{2}, D_{3}, D_{4}, D_{5}\right)=\left(\lambda_{0}+2 \mu_{0}\right)\left(2 D_{1}-D_{1}^{2}\right) \\
& -2 \lambda_{12}^{+}\left(D_{1}, D_{2}, D_{3}\right)-2 \mu_{2}\left(D_{4}, D_{5}\right)+\frac{\left(v_{0}-1\right)}{v_{0}} \lambda_{11}^{-}\left(D_{3}\right)+\left(\lambda_{0}+2 \mu_{0}\right)\left[\left(1-D_{1}\right)^{2}-\left(1-D_{1}\right)^{2}\left(1-D_{2}\right)^{2}\right] \\
& \lambda_{12}^{+}\left(D_{1}, D_{2}, D_{3}\right)=\lambda_{0}\left[\left(1-D_{3}\right)^{2}-\left(1-D_{1}\right)\left(1-D_{2}\right)\left(1-D_{3}\right)\right] \\
& \mu_{2}\left(D_{4}, D_{5}\right)=2 \mu_{0}\left[1-\left(1-D_{4}\right)^{2}\left(1-D_{5}\right)^{2}\right] \\
& \lambda_{22}^{-}\left(D_{2}, D_{3}, D_{4}, D_{5}\right)=\left(\lambda_{0}+2 \mu_{0}\right)\left(2 D_{2}-D_{2}^{2}\right)-2 \lambda_{12}^{-}\left(D_{2}, D_{3}\right) \\
& +\frac{\left(v_{0}-1\right)}{v_{0}} \lambda_{11}^{-}\left(D_{3}\right)-2 \mu_{2}\left(D_{4}, D_{5}\right) \\
& \lambda_{12}^{-}\left(D_{2}, D_{3}\right)=\lambda_{0}\left[\left(1-D_{3}\right)^{2}-\left(1-D_{2}\right)\left(1-D_{3}\right)\right] \\
& \lambda_{11}^{-}\left(D_{3}\right)=\lambda_{0}\left(2 D_{3}-D_{3}^{2}\right)
\end{aligned}
$$


The stress tensor is obtained from the gradient of the elastic potential, as follows:

$$
\sigma(\varepsilon)=\left\{\begin{array}{lll}
\sigma_{-}(\varepsilon)=\nabla_{\varepsilon} \rho \psi_{-}(\varepsilon) & \text { if } & g\left(\varepsilon, D_{T}, D_{C}\right)<0 \\
\sigma_{+}(\varepsilon)=\nabla_{\varepsilon} \rho \psi_{+}(\varepsilon) & \text { if } & g\left(\varepsilon, D_{T}, D_{C}\right)>0
\end{array}\right.
$$

$\sigma_{+}(\varepsilon)=\lambda_{11} \operatorname{tr}(\varepsilon) \mathbf{I}+2 \mu_{1} \varepsilon-\lambda_{22}^{+}\left(D_{1}, D_{2}, D_{3}, D_{4}, D_{5}\right) \operatorname{tr}(\mathbf{A} \varepsilon) \mathbf{A}-\lambda_{12}^{+}\left(D_{1}, D_{2}, D_{3}\right)(\operatorname{tr}(\varepsilon) \mathbf{A}+$

$$
\operatorname{tr}(\mathbf{A} \varepsilon) \mathbf{I})-\lambda_{11}^{-}\left(D_{3}\right) \operatorname{tr}(\varepsilon) \boldsymbol{I}-\frac{\left(1-2 v_{0}\right)}{v_{0}} \lambda_{11}^{-}\left(D_{3}\right)(\boldsymbol{I} \bar{\otimes} \boldsymbol{I}) \varepsilon-\mu_{2}\left(D_{4}, D_{5}\right)(\boldsymbol{A} \varepsilon+\varepsilon \boldsymbol{A})
$$

$\sigma_{-}(\varepsilon)=\lambda_{11} \operatorname{tr}(\varepsilon) \mathbf{I}+2 \mu_{1} \varepsilon-\lambda_{22}^{-}\left(D_{2}, D_{3}, D_{4}, D_{5}\right) \operatorname{tr}(\mathbf{A} \varepsilon) \mathbf{A}-\lambda_{12}^{-}\left(D_{2}, D_{3}\right)(\operatorname{tr}(\varepsilon) \mathbf{A}+\operatorname{tr}(\mathbf{A} \varepsilon) \mathbf{I})$

$-\lambda_{11}^{-}\left(D_{3}\right) \operatorname{tr}(\varepsilon) \boldsymbol{I}-\frac{\left(1-2 v_{0}\right)}{v_{0}} \lambda_{11}^{-}\left(D_{3}\right)(\boldsymbol{I} \bar{\otimes} \boldsymbol{I}) \varepsilon-\mu_{2}\left(D_{4}, D_{5}\right)(\boldsymbol{A} \varepsilon+\varepsilon \boldsymbol{A})$

The constitutive tensor is also obtained from the elastic potential, i. e.:

$$
\mathbf{E}(\varepsilon):=\left\{\begin{array}{lll}
\boldsymbol{E}_{-}(\varepsilon)=\nabla_{\varepsilon}^{2} \rho \psi_{-}(\varepsilon) & \text { if } & g\left(\varepsilon, \boldsymbol{D}_{T}, \boldsymbol{D}_{C}\right)<0, \\
\boldsymbol{E}_{+}(\varepsilon)=\nabla_{\varepsilon}^{2} \rho \psi_{+}(\varepsilon) & \text { if } & g\left(\varepsilon, \boldsymbol{D}_{T}, \boldsymbol{D}_{C}\right)>0,
\end{array}\right.
$$

$\boldsymbol{E}_{+}(\varepsilon)=\boldsymbol{E}_{\boldsymbol{T}}=\lambda_{11}[\boldsymbol{I} \otimes \boldsymbol{I}]+2 \mu_{1}[\boldsymbol{I} \underline{\otimes} \boldsymbol{I}]-\lambda_{22}^{+}\left(D_{1}, D_{2}, D_{3}, D_{4}, D_{5}\right)[\boldsymbol{A} \otimes \boldsymbol{A}]$

$-\lambda_{12}^{+}\left(D_{1}, D_{2}, D_{3}\right)[\mathbf{A} \otimes \mathbf{I}+\mathbf{I} \otimes \mathbf{A}]-\lambda_{11}^{-}\left(D_{3}\right)[\mathbf{I} \otimes \mathbf{I}]-\frac{\left(1-2 v_{0}\right)}{v_{0}} \lambda_{11}^{-}\left(D_{3}\right)[\mathbf{I} \otimes \mathbf{I}]-\mu_{2}\left(D_{4}, D_{5}\right)$

$[\boldsymbol{A} \bar{\otimes} \boldsymbol{I}+\boldsymbol{I} \bar{\otimes} \boldsymbol{A}]$

$\boldsymbol{E}_{-}(\varepsilon)=\boldsymbol{E}_{\boldsymbol{C}}=\lambda_{11}[\boldsymbol{I} \otimes \boldsymbol{I}]+2 \mu_{1}[\boldsymbol{I} \otimes \underline{\otimes} \boldsymbol{I}]-\lambda_{22}^{-}\left(D_{2}, D_{3}, D_{4}, D_{5}\right)\left[\boldsymbol{A} \otimes \boldsymbol{A} \boldsymbol{J}-\lambda_{12}^{-}\left(D_{2}, D_{3}\right)\right.$

$[\mathbf{A} \otimes \mathbf{I}+\mathbf{I} \otimes \mathbf{A}]-\lambda_{11}^{-}\left(D_{3}\right)[\mathbf{I} \otimes \mathbf{I}]-\frac{\left(1-2 v_{0}\right)}{v_{0}} \lambda_{11}^{-}\left(D_{3}\right)[\mathbf{I} \otimes \overline{\mathbf{I}}]-\mu_{2}\left(D_{4}, D_{5}\right)[\boldsymbol{A} \underline{\bar{\otimes}} \mathbf{I}+\mathbf{I} \underline{\otimes} \boldsymbol{A}]$

Taking into account the unilateral effect and assuming that direction 1 be perpendicular to the transverse isotropy local plane in the strain space, the complementary elastic energy of the damaged medium in tension dominant states is now expressed by:

$$
\begin{aligned}
& W_{e+}^{*}=\frac{\sigma_{11}^{2}}{2 E_{0}\left(1-D_{1}\right)^{2}\left(1-D_{2}\right)^{2}}+\frac{\left(\sigma_{22}^{2}+\sigma_{33}^{2}\right)}{2 E_{0}\left(1-D_{3}\right)^{2}}-\frac{v_{0}\left(\sigma_{11} \sigma_{22}+\sigma_{11} \sigma_{33}\right)}{E_{0}\left(1-D_{1}\right)\left(1-D_{2}\right)\left(1-D_{3}\right)}-\frac{v_{0} \sigma_{22} \sigma_{33}}{E_{0}\left(1-D_{3}\right)^{2}} \\
& +\frac{\left(1+v_{0}\right)}{E_{0}\left(1-D_{4}\right)^{2}\left(1-D_{5}\right)^{2}}\left(\sigma_{12}^{2}+\sigma_{13}^{2}\right)+\frac{\left(1+v_{0}\right)}{E_{0}} \sigma_{23}^{2}
\end{aligned}
$$


The variables associated to damage variables in tension with damage activated in previous compression will also be modified, because they are obtained from the elastic potential (20). Therefore, the following relationships are valid:

$$
Y_{T}=\frac{\partial W_{e+}^{*}}{\partial D_{l}}+\frac{\partial W_{e+}^{*}}{\partial D_{4}}=Y_{1}+Y_{4}
$$

$$
Y_{1}=\frac{\sigma_{11}^{2}}{E_{0}\left(1-D_{1}\right)^{3}\left(1-D_{2}\right)^{2}}-\frac{v_{0}\left(\sigma_{11} \sigma_{22}+\sigma_{11} \sigma_{33}\right)}{E_{0}\left(1-D_{1}\right)^{2}\left(1-D_{2}\right)\left(1-D_{3}\right)}
$$

$$
Y_{4}=\frac{\left(1+v_{0}\right)}{E_{0}\left(1-D_{4}\right)^{3}\left(1-D_{5}\right)^{2}}\left(2 \sigma_{12}^{2}+2 \sigma_{13}^{2}\right)
$$

Note that only $Y_{1}$ must to take into account the diffuse damage represented by $D_{2}$ and $D_{3}$. In this case, those damage variables are constants because there is no energy release rates during the damage evolution in tension dominant states related to $D_{2}$ and $D_{3}$. In the case of tension dominant states without activation of damage processes in previous compression, the original version of the damage model is recovered.

It can be verified that the unilateral damage model satisfies two basic requirements of this modeling kind:

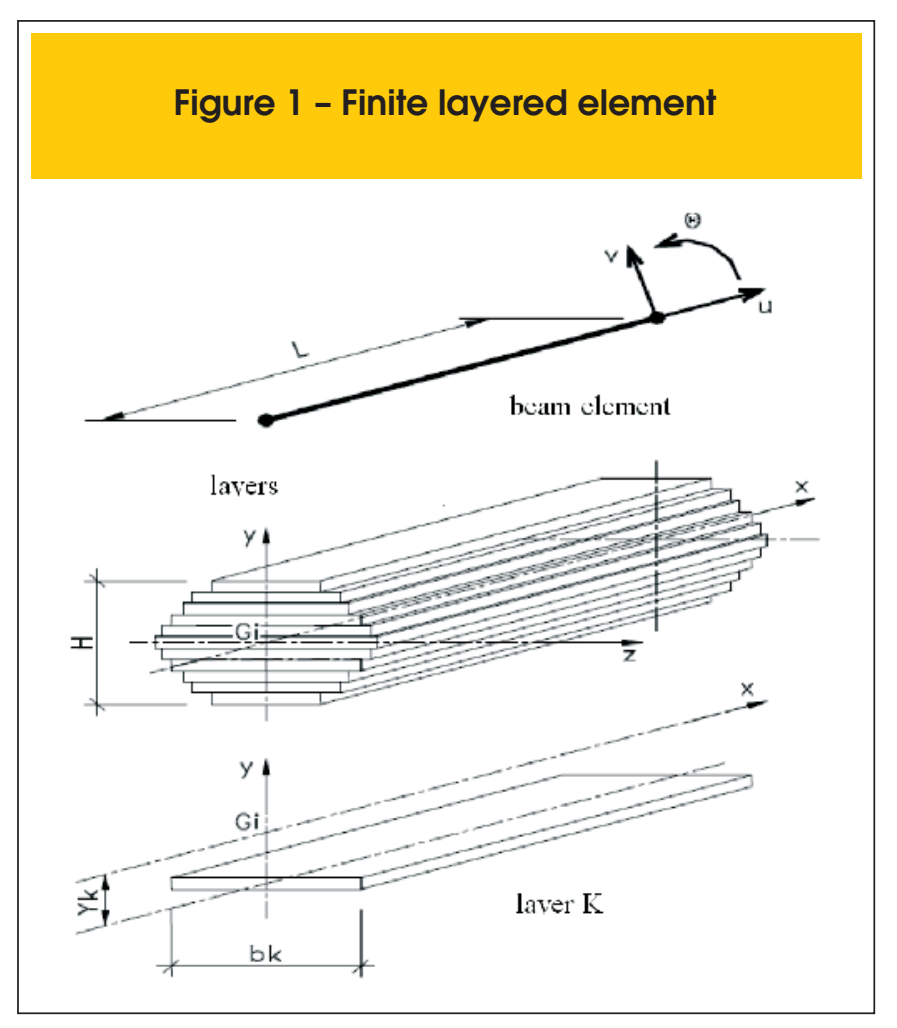

The model does not produce spurious energy dissipation upon closed load paths which do not activate damage, Matallah and La Borderie [19].

The continuity of the stress-strain law across the tension-compression interface is assured (hiperplano $g\left(\varepsilon, \mathbf{D}_{\mathrm{T}}, \mathbf{D}_{\mathrm{C}}\right)$ ), because the damage model is derived from the formulation proposed in Pituba [2], following the requirements of Curnier [1] and Welemane and Comery [10]. The continuity of the stress-strain law between two damage states imposes that the elastic potential must be once continuously differentiable (whole wise), but only piecewise twice continuously differentiable.

Accordingly with Curnier [1], other problem related to this kind of modeling concerns the loss of isotropy of the elasticity tensor in the transition through the tension-compression interface. The isotropy is preserved only if the interface is defined in the same group of symmetry of the elasticity tensor. In the proposed model the hyperplane and elasticity tensor belong to the group of isotropic material if there is not damage process. On the other hand, if there is activation of damage processes, the hyperplane starts to present the symmetry of the transverse isotropic material as well as the elasticity tensor. Anyway, the model always preserves the isotropy of the elasticity tensor.

\subsection{Damage model applied in framed RC structures}

This work intends to show the capabilities of the modified damage model to simulate the mechanical behavior of reinforced concrete structures submitted to reversal loading in possible practical situations of structural engineering. So, it is necessary that the model presents efficient numerical responses, i. e., numerical analyses with low computational cost and a few parameters of the model to be identified. Therefore, the one-dimensional version of the damage model has been implemented in a finite element code for bar structures analysis with finite layered elements in order to model the reinforced concrete framed structures. For the longitudinal reinforcement bars, standard elastoplastic behavior is admitted. In the transversal section, a certain layer can contain steel and concrete, see Fig. 01. A perfect adherence between materials is adopted and an equivalent elasticity modulus and inelastic strain are defined for each layer by using homogenization rule:

$$
E_{k}=\left(1-C_{s k}\right) E_{c k}+C_{s k} E_{s k}
$$

$$
\varepsilon_{i n k}=\left(1-C_{s k}\right) \varepsilon_{c i n k}+C_{s k} \varepsilon_{p s k}
$$

where,

$-C_{s k}$ is the volumetric rate of steel in the layer $\mathrm{N}^{\circ} \mathrm{k}$

$-E_{s k} k$ is the elasticity modulus of steel in the layer $\mathrm{N}^{\circ} \mathrm{K}$

$-E_{c k}$ is the elasticity modulus of concrete in the layer $\mathrm{N}^{\circ} \mathrm{k}$

$-\varepsilon_{\rho s k}$ is the plastic strain of steel in the layer $\mathrm{N}^{\circ} \mathrm{k}$

$-\varepsilon_{\text {ink }}$ is the homogenized inelastic strain in the layer $\mathrm{N}^{\circ} \mathrm{k}$

$-\varepsilon_{\text {cink }}$ is the inelastic strain of concrete in the layer $\mathrm{N}^{\circ} \mathrm{K}$

$-E_{k}$ is the homogenized elasticity modulus in the layer $\mathrm{N}^{\circ} \mathrm{k}$ 
Considering the direction 1 as the longitudinal direction of the finite element, the formulation presented in the previous item is simplified and presented as follows:

$\boldsymbol{E}(\varepsilon):=\left\{\begin{array}{l}\mathbf{E}_{-}(\varepsilon)=\nabla_{\varepsilon}^{2} \rho \psi_{-}(\varepsilon) \text { if } \mathrm{g}\left(\varepsilon, \mathbf{D}_{\mathrm{T}}, \mathbf{D}_{\mathrm{C}}\right)<0, \\ \mathbf{E}_{+}(\varepsilon)=\nabla_{\varepsilon}^{2} \rho \psi_{+}(\varepsilon) \text { if } \mathrm{g}\left(\varepsilon, \mathbf{D}_{\mathrm{T}}, \mathbf{D}_{\mathrm{C}}\right)>0,\end{array}\right.$
$E_{T}=E_{0}\left(1-D_{1}\right)^{2}\left(1-D_{2}\right)^{2}$

$$
E_{C}=E_{0}\left(1-D_{2}\right)^{2}
$$

$$
W_{e+}^{*}=\frac{\sigma_{11}^{2}}{2 E_{0}\left(1-D_{1}\right)^{2}\left(1-D_{2}\right)^{2}}
$$

$$
W_{e-}^{*}=\frac{\sigma_{11}^{2}}{2 E_{0}\left(1-D_{2}\right)^{2}}
$$

$Y_{T}=\frac{\partial W_{e+}^{*}}{\partial D_{1}}=Y_{1}$

$$
Y_{C}=\frac{\partial W_{e-}^{*}}{\partial D_{2}}=Y_{2}
$$

$Y_{1}=\frac{\sigma_{l 1}^{2}}{E_{0}\left(1-D_{1}\right)^{3}\left(1-D_{2}\right)^{2}}$

$$
Y_{2}=\frac{\sigma_{11}^{2}}{E_{0}\left(1-D_{2}\right)^{3}}
$$

The one-dimensional version of the model takes into account permanent strains induced by damage evolution. Assuming, for sim- plicity, that the permanent strains are composed exclusively by volumetric strains, as it has already been considered in Comi [7], and taking into account the unilateral effect, the evolution law results:

$$
\dot{\varepsilon}^{p}=\left(\frac{\beta_{1}}{\left(1-D_{1}\right)^{2}} \dot{D}_{1}+\frac{\beta_{2}}{\left(1-D_{2}\right)^{2}} \dot{D}_{2}\right) \mathbf{I}
$$

Observe that $\beta_{1}$ and $\beta_{2}$ are parameters directly related to the evolutions of permanent strains induced by damage in tension and in compression, respectively. The consideration of the permanent strains improves the capture of the transverse strains by the model, as it can see in Pituba and Fernandes [4]. Besides, the model predicts the change in sign of the volumetric strain.

\section{Micromechanical theory}

Although the damage model has been based on the macromechanical behavior of the concrete, this item intends to show the strong connection between the model and the micromechanical theory. The description of the damage activation-deactivation process as part of macroscopic modeling requires knowing when the transition between these two states of damage occur and how damage deactivation affect the elastic properties of the material, Welemane and Comery [10]. Moreover, there is a difficulty in recognizing tension and compression states in 3D micro-scale analysis in order to adopt a differentiable Gibbs potential. It is noted that the formulation for bimodular anisotropic damaged media proposed in Pituba [2] replies the first question (see Equation (7)). Besides, the continuity of the stress-strain law has been assured. In this context, this section aims to point out the influence of the opening-closure of microdefects on the elastic properties of the microcracked concrete.

Following Welemane and Comery [10], consider a RVE (representative volume element) of an homogeneous isotropic elastic linear matrix (Young modulus $\mathrm{E}_{0}$ and Poisson rate $\mathrm{v}_{0}$ ) weakened by an array of $\mathrm{N}$ randomly distributed flat penny-shaped microcracks (unit normal $n_{k}$, radius $a_{k}$ ), whose radii are very small in comparison with

\section{Figure 2 - Parallel microcracks on concrete submitted to uniaxial tension stress}

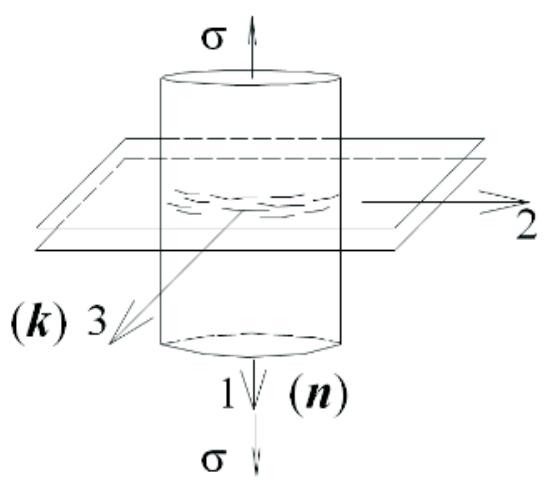


the size of the RVE. Assuming non-interaction among microcracks and sliding without friction of their lips, the free enthalpy of the microcracked medium is given by:

$$
\begin{aligned}
& \mathrm{u}=\frac{\left(1+\mathrm{v}_{0}\right)}{2 E_{0}} \operatorname{tr}(\sigma \cdot \sigma)-\frac{\mathrm{v}_{0}}{2 E_{0}}(\operatorname{tr\sigma })^{2}+\frac{8}{3 V} \frac{\left(1-v_{0}^{2}\right)}{\left(2-v_{0}\right) E_{0}} \sigma: \sum_{K=l}^{N} a_{K}^{3} \\
& {\left[n_{k}^{\otimes 2} \bar{\otimes}_{-} I+I \otimes n_{k}^{\otimes 2}-\left[2-\left(2-v_{0}\right) H\left(\sigma_{n}^{k}\right)\right] n_{k}^{\otimes 4}\right]: \sigma}
\end{aligned}
$$

The Heaviside function $\mathrm{H}$ depending on the normal stress to each microcrack is open $\left(\sigma_{n}^{\mathrm{k}} \geq 0\right)$ or closed $\left(\sigma_{n}^{\mathrm{k}}<0\right)$.

Consider the simple case of a material weakened by a single array of parallel microcracks with unit normal $\mathrm{n}$ as in Fig. 2 and parameter $\mathrm{A}=16\left(1-v_{0}^{2}\right) /\left(6-3 v_{0}\right)$. This case is interesting for the damage model proposed in this work because the effective medium exhibits the symmetry associated with the geometric shape of the microcracks with the privileged direction $\mathrm{n}$ (transverse isotropic material).

Then, the elastic moduli are fully determined by five independent coefficients $E(\mathbf{n}), E(\mathbf{t}), v(\mathbf{n}, \mathbf{t}), v(\mathbf{t}, \mathbf{k})$ and $\mu(\mathbf{n}, \mathbf{t})$, for any vectors $\mathbf{t}$ and $\mathbf{k}$ forming with $\mathrm{n}$ an orthonormal basis of $\mathrm{R}^{3}$. Using $\mathrm{Eq}(46)$, it can be obtained the elastic moduli mentioned above.

$$
E(n)=E_{0}\left[1+\frac{A}{V} \sum_{K=1}^{N} a_{k}^{3}\left(2-v_{0}\right) H\left(\sigma_{n}^{k}\right)\right]^{-1}
$$

$$
v(n, t)=v_{0}\left[1+\frac{A}{V} \sum_{K=1}^{N} a_{k}^{3}\left(2-v_{0}\right) H\left(\sigma_{n}^{k}\right)\right]^{-1}
$$

$E(\boldsymbol{t})=E_{0}$

$$
v(\boldsymbol{t}, \boldsymbol{k})=v_{0}
$$

$$
\mu(n, t)=\mu_{0}\left[1+\frac{A}{\left(1+v_{0}\right) V} \sum_{K=1}^{N} a_{k}^{3}\right]^{-1}
$$

In Welemane and Comery [10] are described some conclusions about eqs (47)-(51) that are useful for a discussion about the proposed model. In general way, a macroscopic approach of the unilateral effect in brittle materials should no longer be considered only by the single restoration of the Young modulus in the direction normal to closed microcracks. Therefore, based on micromechanical observations, some important aspects related to unilateral effect of damage processes can be pointed out:

- The elastic moduli $E(\mathbf{n})$ and Poisson ratio $v(\mathbf{n}, \mathbf{t})$, related to normal direction to parallel microcracks, are affected by the evolution of the microdefects. In particular, those moduli recover their initial values $\left(E_{0}\right.$ and $\left.v_{0}\right)$ when the microcracks are closed.

- In the other hand, the shear modulus $\mu(\mathbf{n}, \mathbf{t})$ remains the same when the microcracks are closed (partial deactivation of damage). This behavior is consistent with the hypothesis about tangential jump null of the constitutive tensor. However, the elastic moduli $E(\mathbf{m}), v(\mathbf{m}, \mathbf{p})$ and $\mu(\mathbf{m}, \mathbf{p})$ related to directions with different orientations at principal axes $(\mathbf{n}, \mathbf{t}$ and $\mathbf{k})$ are partially recovered when the microcracks close.

The particular nature of the microdefects contribution allows extending these considerations for any of $N$ microcracks with different normal vectors. In this context, let us compare the damaged elastic moduli given by the proposed model to those ones given by the micromechanical equations. Then, considering Fig. 2 and assuming, for instance, that the transversal isotropy local plane is coincident with the 2-3 plane, the elastic moduli given by the proposed model in dominant compression (subscript $C$ ) and in tension (subscript $T$ ) regimes are written as:

$E_{T 1}=E_{0}\left(1-D_{1}\right)^{2}\left(1-D_{2}\right)^{2} ; E_{C l}=E_{0}\left(1-D_{2}\right)^{2} \quad$ (52)

$$
v_{T 12}=v_{T 13}=v_{0} \frac{\left(1-D_{1}\right)\left(1-D_{2}\right)}{\left(1-D_{3}\right)} ; v_{C 12}=v_{C 13}=v_{0} \frac{\left(1-D_{2}\right)}{\left(1-D_{3}\right)}
$$

$E_{T 2}=E_{T 3}=E_{0}\left(1-D_{3}\right)^{2} ; E_{C 2}=E_{C 3}=E_{0}\left(1-D_{3}\right)^{2}$

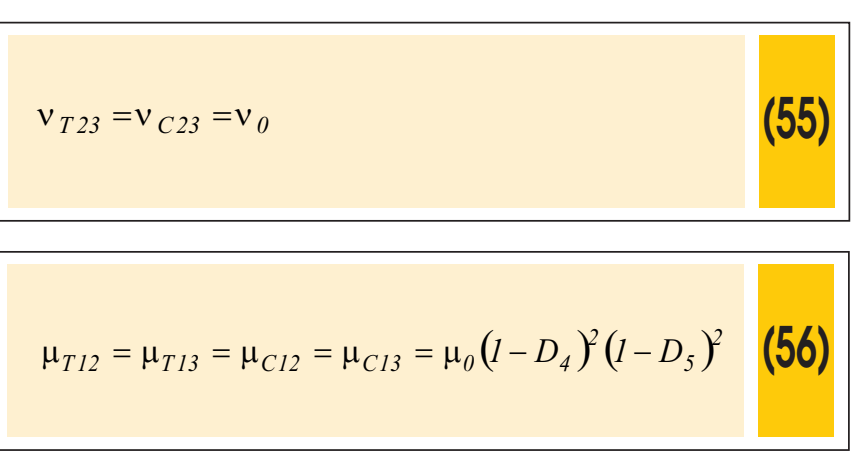

The longitudinal elastic moduli in tension and in compression in the direction 1 depend on the dominant state, i. e., of the openingclosure criterion. This is also valid for the Poisson ratio in the 12 and 13 planes. On the other hand, the Poisson ratio in the 


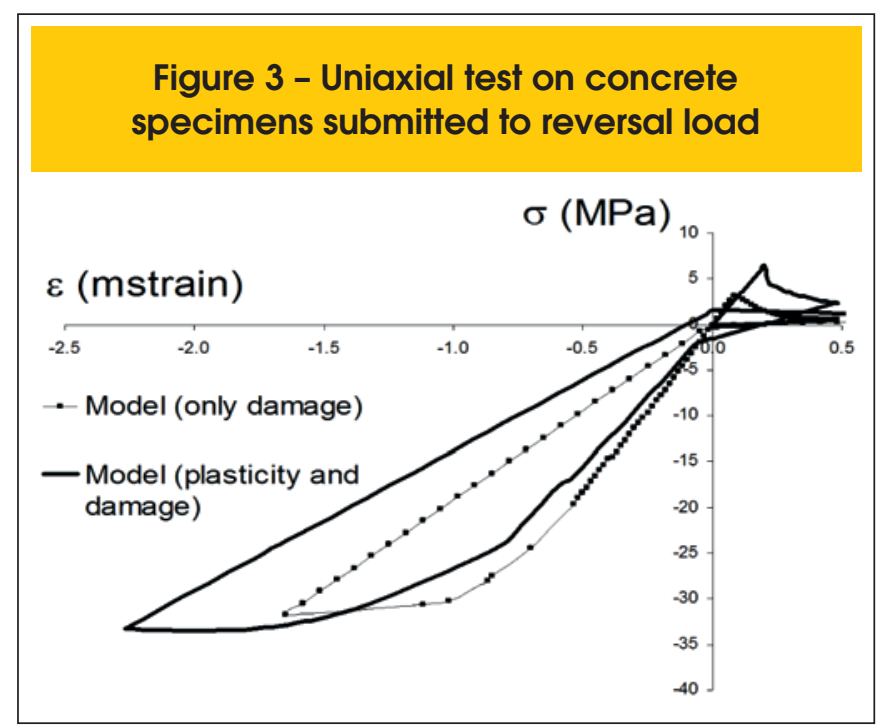

23 plane (transversal isotropy local plane) is not affected by the damage process. The shear moduli are not changed in the transition from the tension to compression regimes and vice-versa. Observe Eq. (54) and consider the transition from dominant tension regime (damage process in tension activated or not) to the compression regime without previous compression. In this case one has: $\mathrm{E}_{\mathrm{T} 2}=\mathrm{E}_{\mathrm{T} 3}=\mathrm{E}_{\mathrm{C} 2}=\mathrm{E}_{\mathrm{C} 3}=\mathrm{E}_{0}$. This result is in correspondence with the form described by (49). Indeed, the $\left(1-D_{3}\right)^{2}$ coefficient is necessary to take into account the diffuse damage in previous compression when the current dominant state is tension.

Obviously, in general cases, when the damage process is activated, the formulation starts to involve the tensor $\mathbf{A}$, which depends on the knowledge of the normal to the transverse isotropy plane,

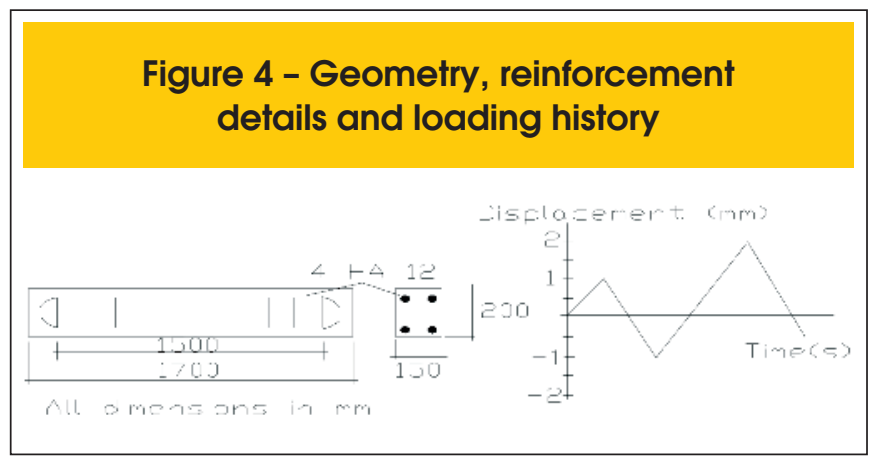

Pituba and Fernandes [4]. Therefore, the discussion about elastic moduli presented above is valid but that moduli are dependents of the tensor $\mathbf{A}$, as described in item 2.1.

Finally, it is observed that despite the proposed model has macromechanical motivations in the macroscopic behaviour of the concrete, the model assists to the requirements suggested by Welemane and Comery [10] for the micromechanical analysis of the unilateral effect in materials.

\section{Numerical applications}

Initially, the unilateral model is used in the simulation of an uniaxial test in concrete specimens subject to reversal load in order to show the qualitative numerical response. Observe that the permanent strains are important in the definition of the hyperplane, in the sense that the total strains start to compose the criterion, Eq. (8). The initial stiffness recovery can be clearly observed taking into account permanent strain in the dominant tension regime. It is noted the contribution of the diffuse damage generated in previous compression regimes when dealing to tension regimes.

\section{Figure 5 - Parametric identification in uniaxial compression test - La Borderie's RC Beam}

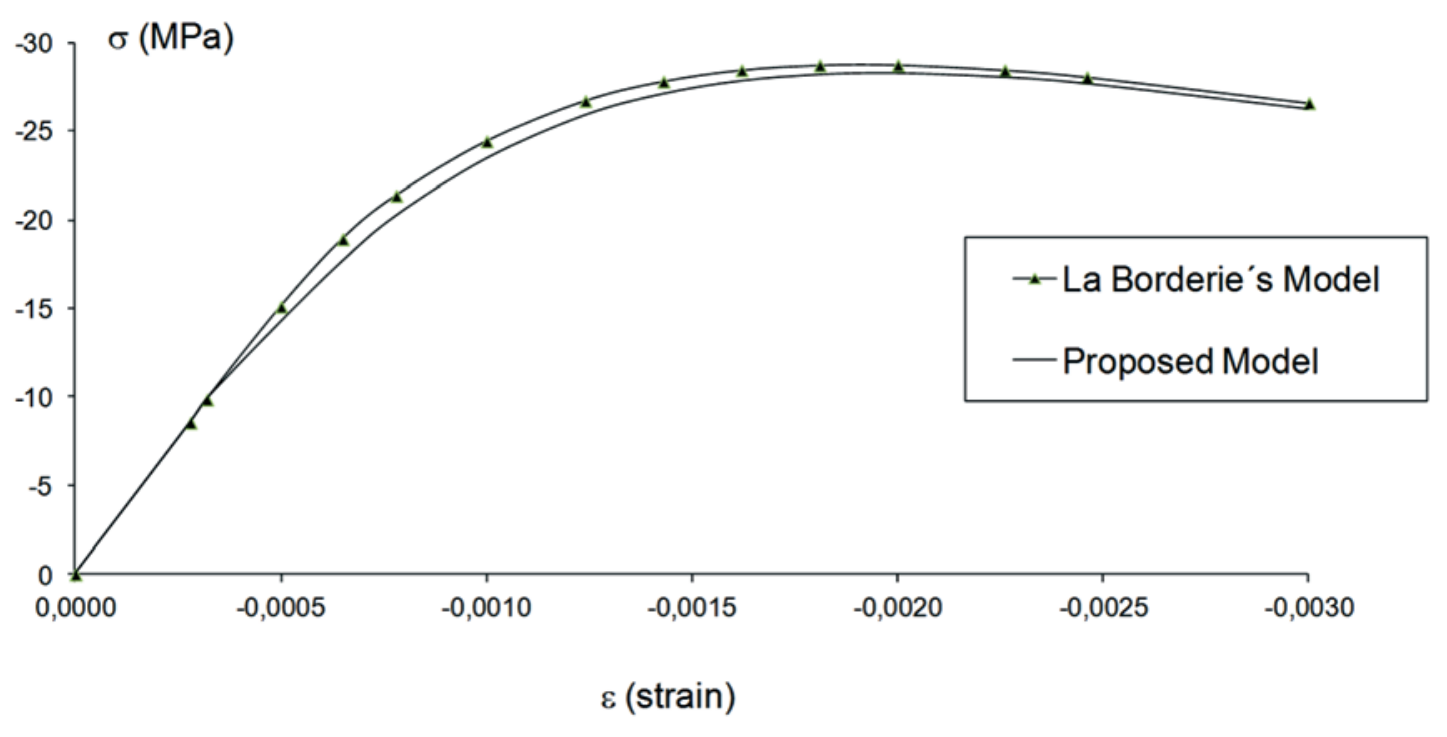




\section{Figure 6 - Parametric identification in uniaxial tension test - La Borderie's RC Beam}

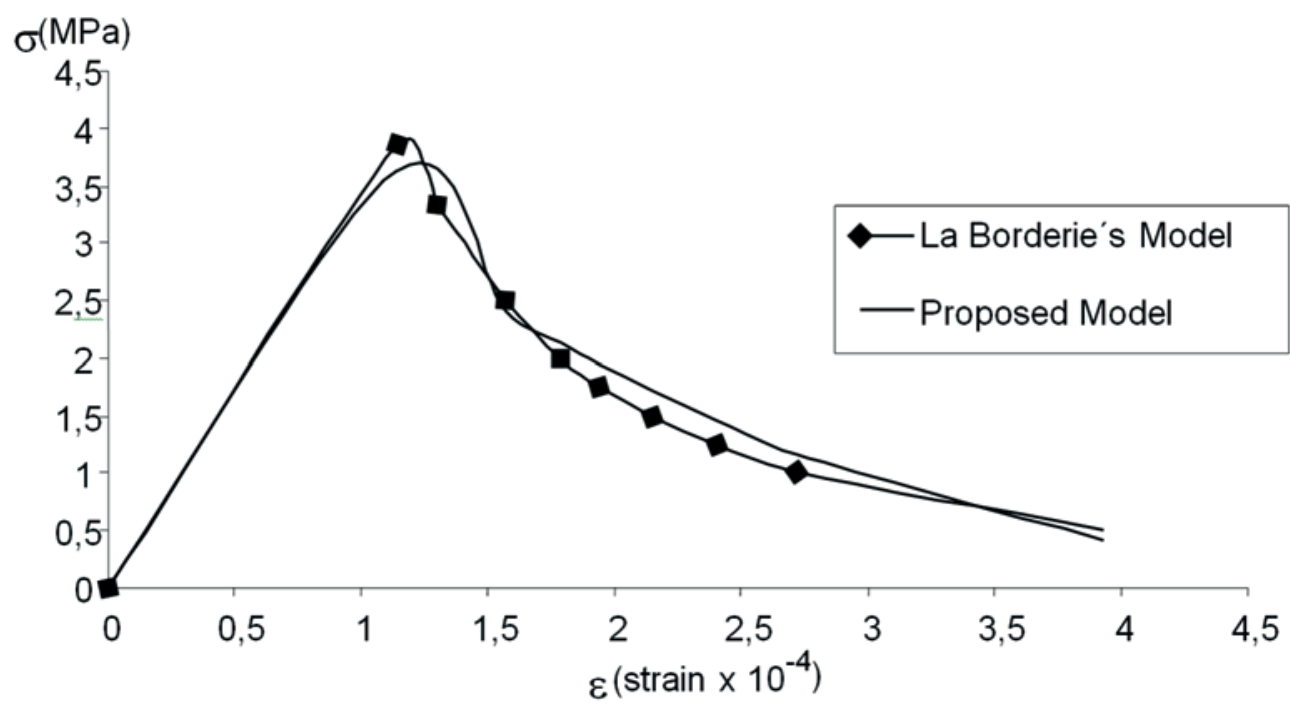

\subsection{Reinforced concrete beam with symmetric reinforcement}

This example deals with a test performed by La Borderie [20] and Matallah and La Borderie [19] that corresponds to a reinforced concrete beam in a configuration of three points cyclic flexion. The beam is subject to cyclic loading at the middle of the span. The concrete has elasticity modulus $\mathrm{E}_{\mathrm{c}}=31,800 \mathrm{MPa}$; the steel has $\mathrm{E}_{\mathrm{s}}$ $=210,000 \mathrm{MPa}$, yielding stress of $445 \mathrm{MPa}$ and ultimate stress of $540 \mathrm{MPa}$. In the experimental test, the beam is subjected to two loading cycles of amplitude, the first one is $1 \mathrm{~mm}$ and the second one $2 \mathrm{~mm}$ (see Fig. 4). The beam geometry and its reinforcement distribution are illustrated in Fig. 4.

Figures 5 and 6 show the results of the parametric identification of the proposed damage model. The parameters used by La Borderie [20] were taken as reference in the simulation of uniaxial tension and compression tests. Table 1 presents the parameter values. It is important to note that the experimental tests do not present loading/unloading paths to identify $\beta_{1}$ and $\beta_{2}$. Therefore, the parameters $\beta_{1}$ and $\beta_{2}$ have been adopted in order to obtain the permanent strains evidenced by the numerical analysis of the concrete beam during the unloading process. This adoption has been made without interference in the value of the compression and tension strengths of the concrete.

In the numerical analysis, displacements increments have been enforced in the middle of the span. Using the advantage of symmetry, only half of the beam has been discretized into 20 finite elements. The transversal sections were divided into 16 layers where the reinforcement layers are located in the medium planes of the second and fifteenth layers. In Fig. 7 are shown the numerical and experimental responses of the vertical force and displacement in the middle of the span related to the first stage of the loading. It is noted the good precision of the numerical response.
In the other hand, in the Fig. 8 is illustrated the global response of whole test. The results obtained by the model are satisfactory despite the limited parametric identification of the parameters related to permanent strains. The ultimate experimental loads related to the first and second cycles are obtained by the both analyses: when only damage processes are considered and the other one when permanent strains $\left(b_{1}\right.$ and $\left.b_{2}\right)$ are taken into account too. However, the permanent strains in the unloading processes are only captured by the modelling with permanent strains, as expected. In general way, the model reproduces satisfactorily the cyclic behaviour of the beam.

Besides, the damage profile is also close to experimental test observations, see Matallah and La Borderie [19]. In Fig. 09 is shown the damage distribution in tension regimes at two points of the curve illustrated in Fig. 08. The first point is located at the end of the first loading and the second one is located in the end of the second loading (reversal loading). These distributions have shown the opening/closure cracks process. The first damage in tension zone $\left(D_{1}\right)$ occurs in the bottom of the beam. On the other hand, when the load is inverted, the damage in tension zone $\left(D_{1}\right)$ appears in the upper zone of the beam, but the $D_{1}$ distribution in the bottom

\section{Table 1 - Parameters for the proposed damage model - La Borderie's RC Beam}

\begin{tabular}{cc} 
Tension & Compression \\
$\mathrm{Y}_{01}=6.0 \times 10^{-5} \mathrm{MPa}$ & $\mathrm{Y}_{02}=3.0 \times 10^{-3} \mathrm{MPa}$ \\
$\mathrm{A}_{1}=-0.93$ & $\mathrm{~A}_{2}=1.50$ \\
$\mathrm{~B}_{1}=110 \mathrm{MPa}^{-1}$ & $\mathrm{~B}_{2}=10.01 \mathrm{MPa}^{-1}$ \\
$\beta_{1}=8 \times 10^{-5} \mathrm{MPa}$ & $\beta_{2}=1.0 \times 10^{-3} \mathrm{MPa}$ \\
\hline
\end{tabular}


Figure 7 - Experimental and numerical responses - first loading

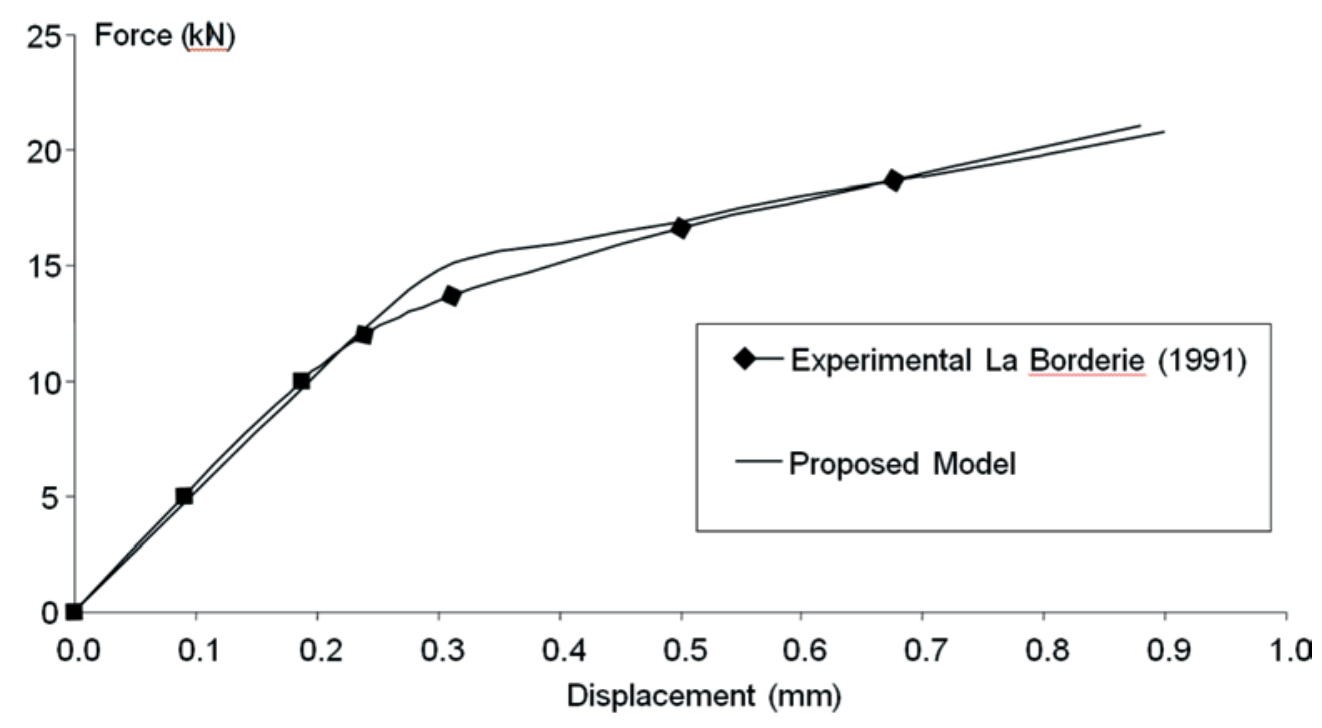

of the beam remains the same, although there is no increasing of values. Therefore, the cracks previously open are now closed. Note that the damage processes in the compression regimes $\left(D_{2}\right)$ are not so important in this numerical application, according to observations in La Borderie [20]. It can be observed that the symmetric arrangement of the reinforcement leads to an additional support to compression stresses in the concrete.

\subsection{Reinforced concrete frame}

This experimental test was originally performed by Vecchio and Emara [21] taking into account just proportional loading/unloading, but without reversal loading. However, in this work, the reinforced concrete frame is submitted to loading/unloading and then a reversal loading is applied in order to show the potentialities of

\section{Figure 8 - Global response of the reinforced concrete beam}

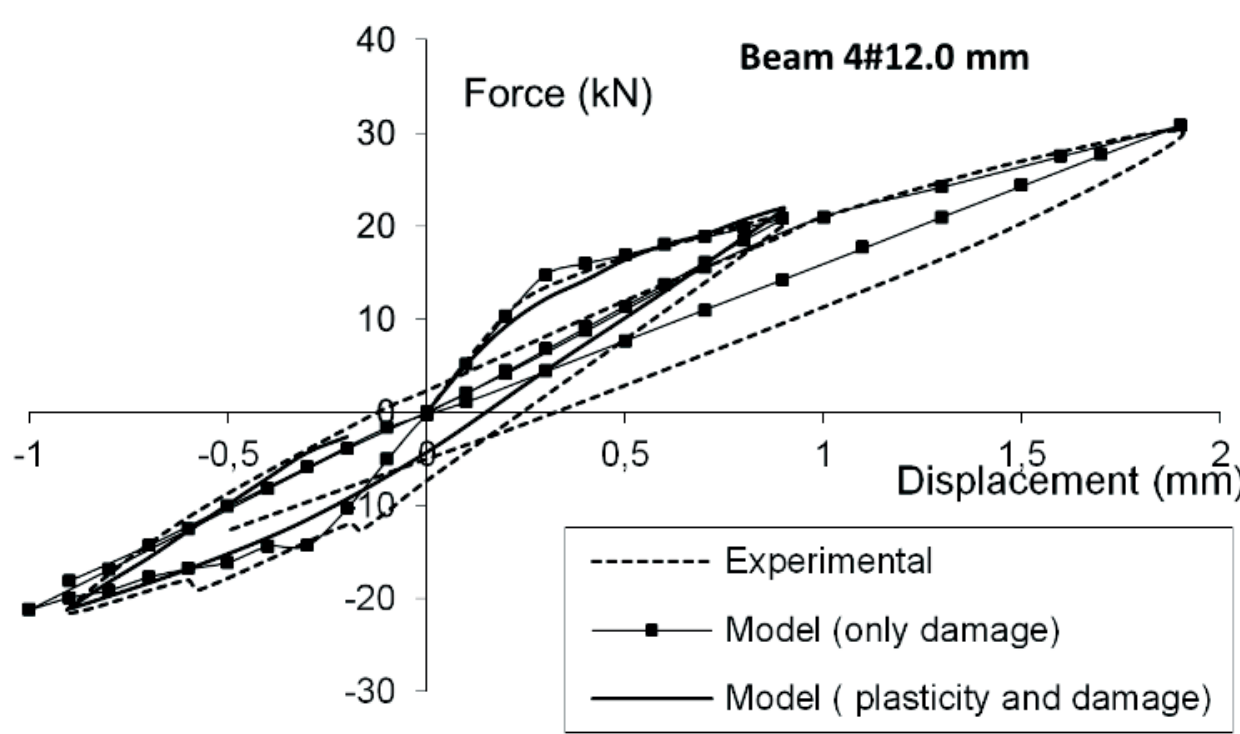




\section{Figure 9 - Damage distribution in tension $\left(D_{1}\right)$ : a) End of first loading; b) End of second loading (reversal loading)}

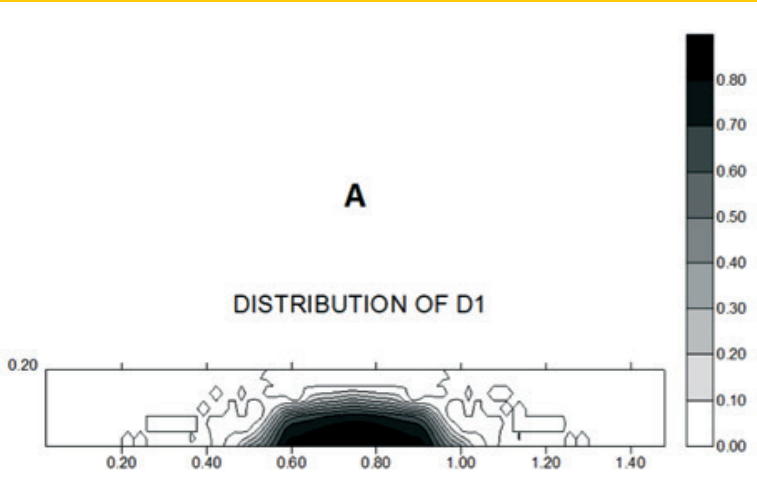

the proposed model to simulate the collapse of frames in cyclic loading conditions.

The frame geometry and its reinforcement distribution are illustrated in Fig. 10. The concrete has the elasticity modulus $E_{c}=30,400$ $\mathrm{MPa}$ and the steel has $\mathrm{E}_{\mathrm{s}}=192,500 \mathrm{MPa}$, yielding stress of 418 $\mathrm{MPa}$ and ultimate stress of $596 \mathrm{MPa}$. For the mechanical behaviour of the reinforcement, a bilinear elastoplastic model has been adopted with a reduced elasticity modulus in the second branch $\left(E_{s 2}=0.009 E_{s}\right)$. Also, Table 2 contains the values for the concrete parameters as well as in the Fig. 11 is illustrated the parametric identification by fitting experimental curve on compression test given in Vecchio and Emara [21]. However, the parameters in tension regime have been obtained using the La Borderie's model response given by Pituba [3].

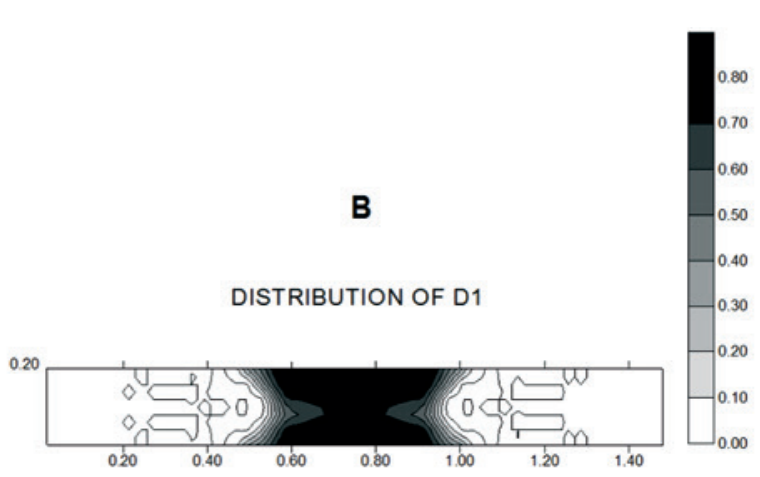

Two kinds of support conditions have been used for the numerical analyses. The first one, Case 1, considers the frame clamped on two columns. The second one, case 2 , considers the frame with a support beam. The goal is to investigate the possible influence of the support conditions in the analyses performed here.

In the experimental test, initially it has been applied an axial load of $700 \mathrm{kN}$ at each column, which was maintained constant throughout the test. The lateral force has been applied in increments up to the frame ultimate loading be achieved. In the numerical analysis originally performed by Pituba [3], displacements increments have been enforced in the application point of the horizontal force up to the frame ultimate load. In that work, it has been performed loading and unloading trying to simulate the experimental behaviour of the frame. The numerical results were very satisfactory simulating

\section{Figure 10 - Geometry and reinforcement details of the frame}
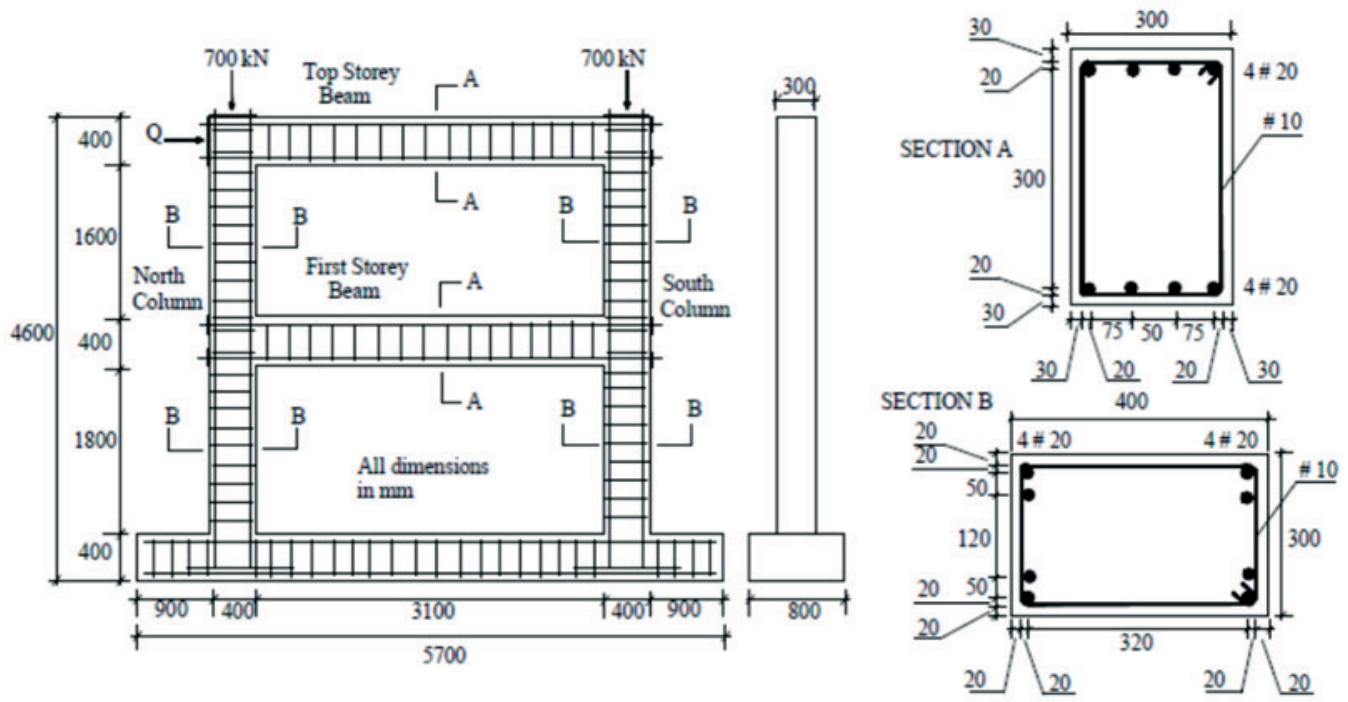


\section{Figure 11 - Parametric identification: a) Uniaxial compression test; b) Uniaxial tension test}
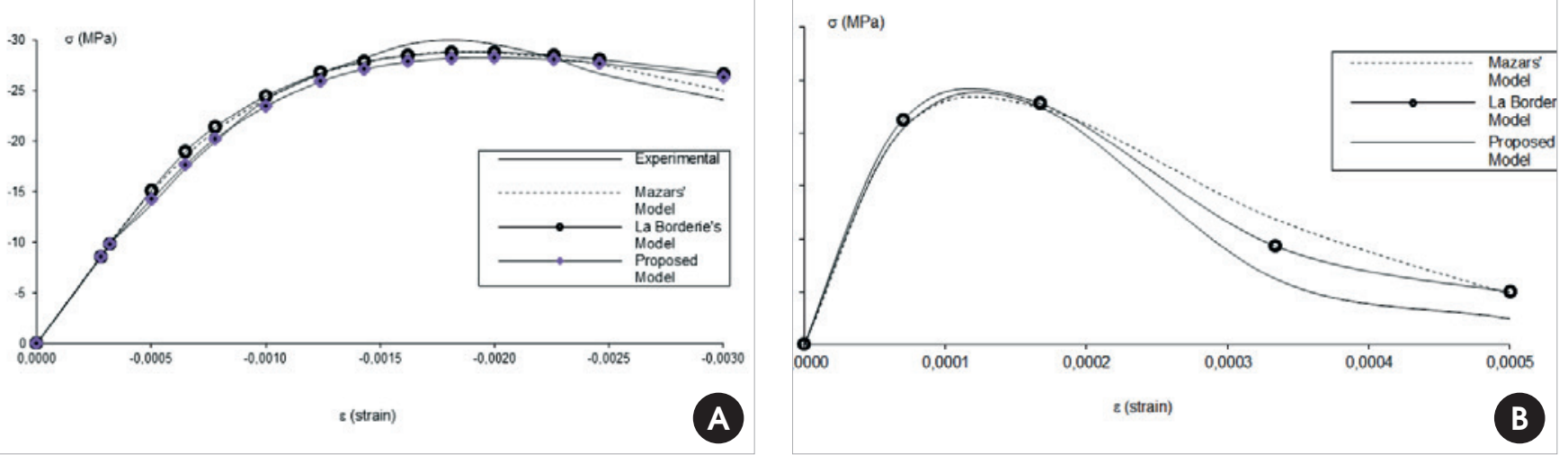

the ultimate load as well as the residual strains.

For the Case 1, the frame has been discretized into 30 finite elements, 10 of which have been used in the discretization of each column and 5 in each beam. The transversal sections have been divided into 10 layers. For the Case 2, the same discretization for the Case 1 has been used with addition of 14 finite elements to model the support beam. In order to investigate the potentialities of the improvement of the damage model proposed in section 2.2, the framed structure has been analyzed

\section{Table 2 - Parameters values of the proposed damage model - RC Frame}

\begin{tabular}{cc} 
Tension & Compression \\
$\mathrm{Y}_{01}=0,72 \times 10^{-4} \mathrm{MPa}$ & $\mathrm{Y}_{02}=0,17 \times 10^{-2} \mathrm{MPa}$ \\
$\mathrm{A}_{1}=49$ & $\mathrm{~A}_{2}=0,30$ \\
$\mathrm{~B}_{1}=6560 \mathrm{MPa}^{-1}$ & $\mathrm{~B}_{2}=5,13 \mathrm{MPa}^{-1}$ \\
$\beta_{1}=1 \times 10^{-6} \mathrm{MPa}$ & $\beta_{2}=1 \times 10^{-3} \mathrm{MPa}$ \\
\hline
\end{tabular}

attempting to perform an unloading of the horizontal force $Q$, including reversal loading. The goal is to observe the consistency of the qualitative response provided by the damage model. The numerical and experimental responses are illustrated in Fig. 13 for the both cases of support conditions, where the graphs represent the applied horizontal force versus horizontal displacement computed at the superior floor of the frame (see Fig. 10). Note that the numerical responses for the both support conditions are quite similar. In fact, the damage

Figure 12 - Mesh and Boundary conditions used for the analyses

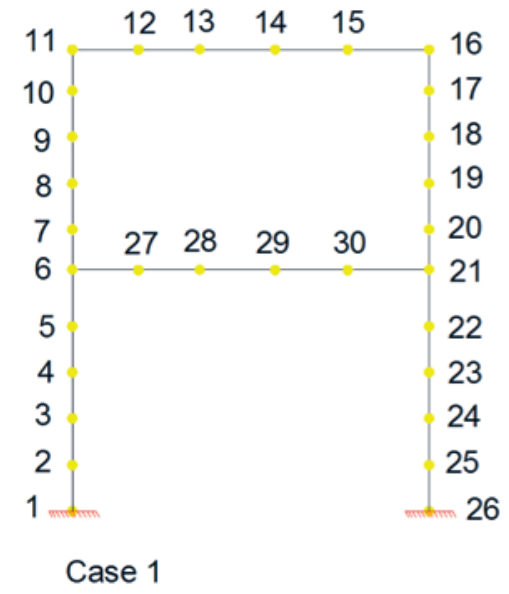

Case 1

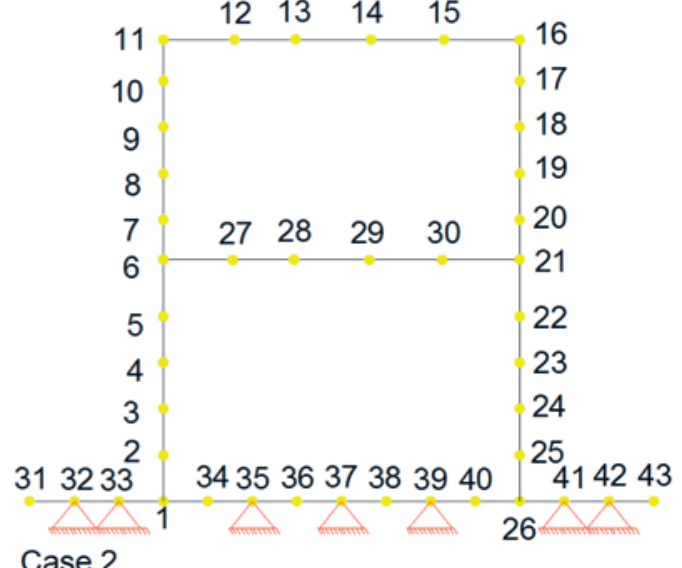

Case 2 
model does not consider the concrete strength complementary mechanisms for the mechanical behaviour of the framed structure, such as: aggregate interlock and dowel action. Following Vecchio and Emara [21] and Nogueira et al. [22], the shear contribution is important for this structure leading to different numerical responses as evidenced in Nogueira et al. [22].

It can be noted the agreement between numerical and experimental responses during the unloading process. In fact, it evidences the good performance of the damage model to capture residual strains. In this stage, the loading capacity of the frame has been achieved and the damage level is high in most zones of the frame, as it can see in Figure 14 for the Case 1. Note that the figure presents the damage distribution related to tension regimes $\left(D_{1}\right)$ because the analysis has shown the importance of that variable. This is related to the concept of the damage model proposed in this work. It is possible to observe the evolution of the damage processes within the stages displayed in Fig. 14

Besides, in Fig. 13 the symmetric behaviour of the frame related to load capacity when the horizontal force $Q$ is applied to right direction and then it has been changed to left direction. In the first case, the load level capacity is about $294.3 \mathrm{kN}$. On the other hand, the load level capacity is $286.6 \mathrm{kN}$ for the second case. Note yet, the capability of the model to simulate the recovery of the load capacity when the first cycle of loading is complete.

There are some parts of the frame with high values of damage variable $D_{1}$ that together with the yielding of the reinforcement bars contribute to concentrate damage-plastic zones like plastic joints. It can be observed these zones in first and second floor beam/column junctions and, mainly, in the supports of the frame. These observations are in agreement with described in Vecchio and Emara [21].

\subsection{Reinforced concrete beam with unsymmetrical reinforcement}

The third numerical application is about a reinforced concrete beam with unsymmetrical reinforcement. This numerical application has been originally performed by Pituba and Lacerda [5], but only monotonic loading has been imposed to the beam in that work. The elastic parameters of the concrete are $f_{c}=25 \mathrm{MPa}$ and $E_{c}=32.3 \mathrm{MPa}$. For the reinforcement has been adopted $E_{s}=205$ $\mathrm{GPa}$, yielding stress $590 \mathrm{MPa}$ and ultimate stress $750 \mathrm{MPa}$. The geometric characteristics of the beam are given in Figure 15. The loading is composed by two equal forces applied on the beam.

Table 3 presents the values of the parameters used in the analysis and adopted from Pituba and Lacerda [5], however in this work is considered the plastic strains generated by the damage model.

The structure has been discretized into 16 finite elements and the transversal sections have been divided into 15 layers where 3 layers have been used to represent the reinforcement bars according with Fig. 15. The numerical and experimental responses are illustrated in Fig. 16. In the first loading, it is noted that the numerical results are very close to the experimental ones evidencing a good quality response in the sense that captures the history of the mechanical behaviour of the structure. In this work, the numerical analysis continues with the unloading process about $110 \mathrm{kN}$, where the beam is quite damaged in tension zone (bottom of the beam) and the reinforcement bars present evident yielding in the same zone, Pituba and Lacerda [5]. The value to start the unloading process $(110 \mathrm{kN})$ has been adopted in order to overcome numerical problems related to high values of the damage variable in tension.

The unloading process modelled by the damage model presents very important qualitative results. The damage model can

\section{Figure 13 - Numerical and experimental results of reinforced concrete frame}

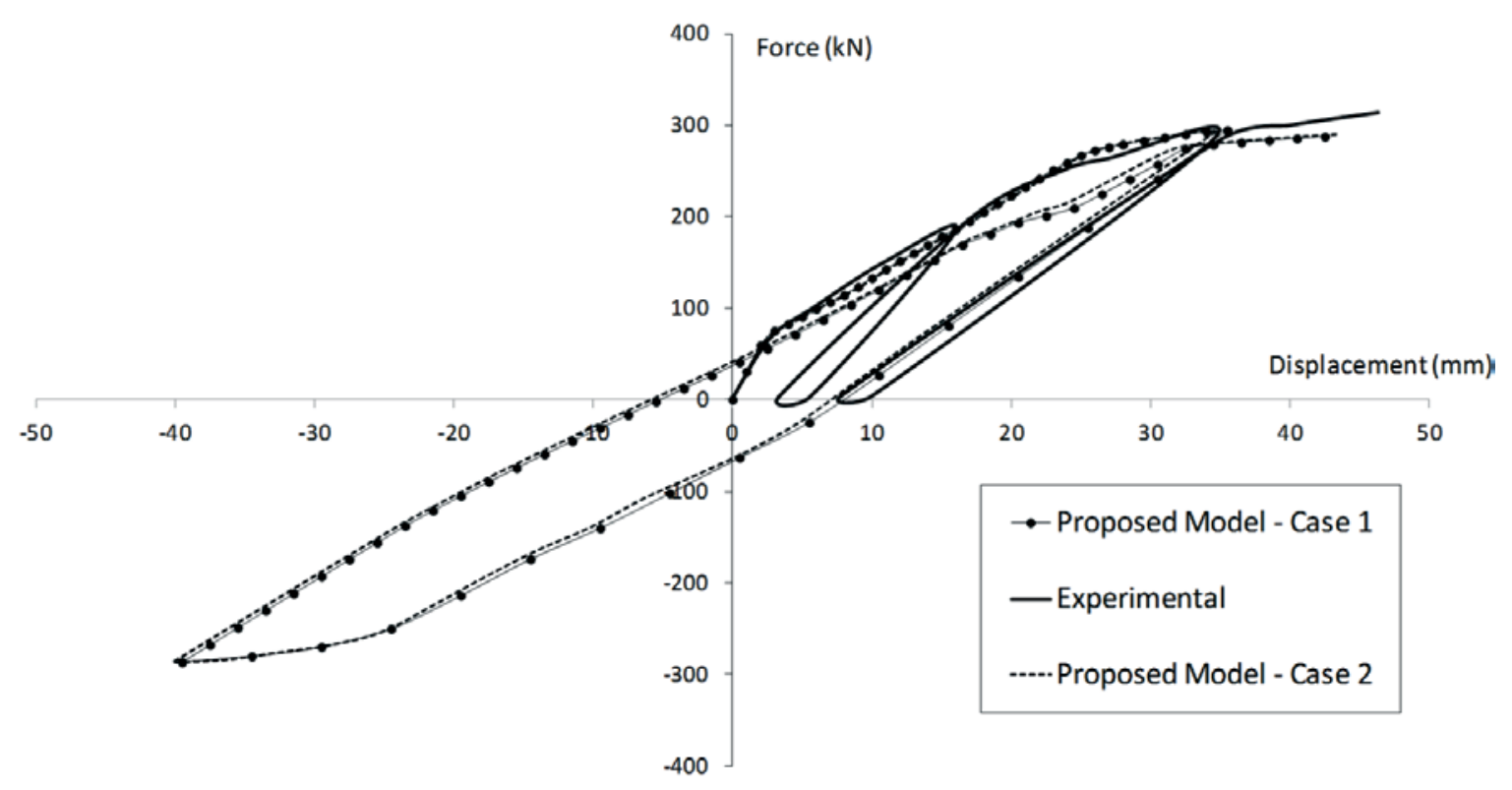




\section{Figure 14 - $D_{1}$ damage distribution: a) First loading $(Q=59.3 \mathrm{kN})$; b) First loading $(Q=75.6 \mathrm{kN})$; c) First loading $(Q=294.3 \mathrm{kN})$; d) Reversal loading $(Q=-286.6 \mathrm{kN})$, see Fig. 12}

DISTRIBUTION OF D1

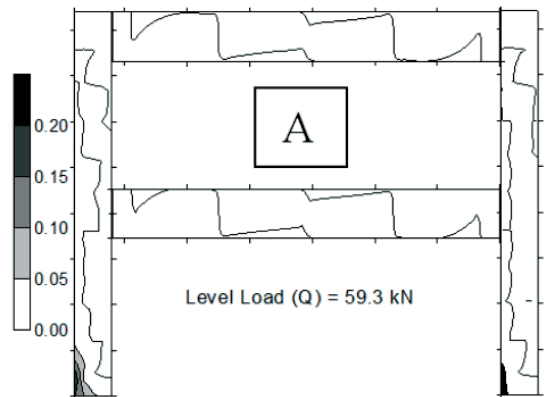

DISTRIBUTION OF D1

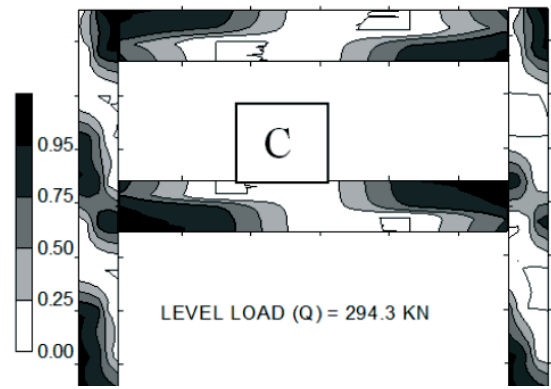

simulate a residual displacement when the reverse loading takes place. Furthermore, it is observed that due to the asymmetric arrangement of the reinforcement, i. e., there is sufficient reinforcement at the bottom to resist the tension stresses in the first loading and insufficient reinforcement $(2 \# 6.3 \mathrm{~mm})$ on the upper zone to resist the tension stresses in that zone when the load is changed. In this situation, the structure experiences a damage process in tension very intense in the upper zone of the beam. Therefore, it is natural that the strength of the beam be much smaller than in the initial first loading. It can be observed that the concrete does not have strength to the applied force and only the reinforcement resists to tension stresses indicating a strong plastic strain.
DISTRIBUTION OF D1

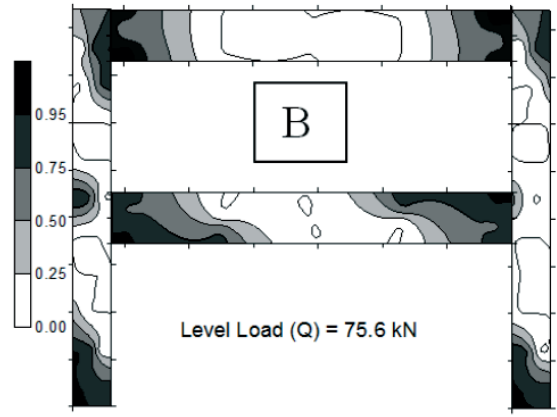

DISTRIBUTION OF D1

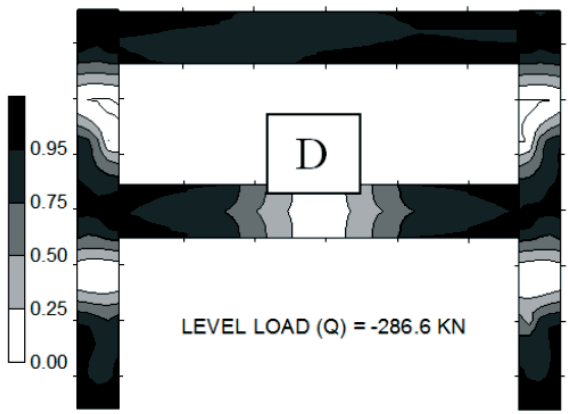

\section{Conclusions}

In this work, an improvement of a damage model incorporating the capability to simulate the unilateral effect of the concrete has been presented. This paper has shown that the proposed damage model assists to the requirements suggested by Welemane and Comery [10] for the micromechanical analysis of the unilateral effect in materials. Besides, the continuity of the stress-strain law across the tensioncompression interface has been assured and the model always preserves the isotropy of the elasticity tensor.

In order to validate the proposed model in practical situations, a

\section{Figure 15 - Geometry and reinforcement details}
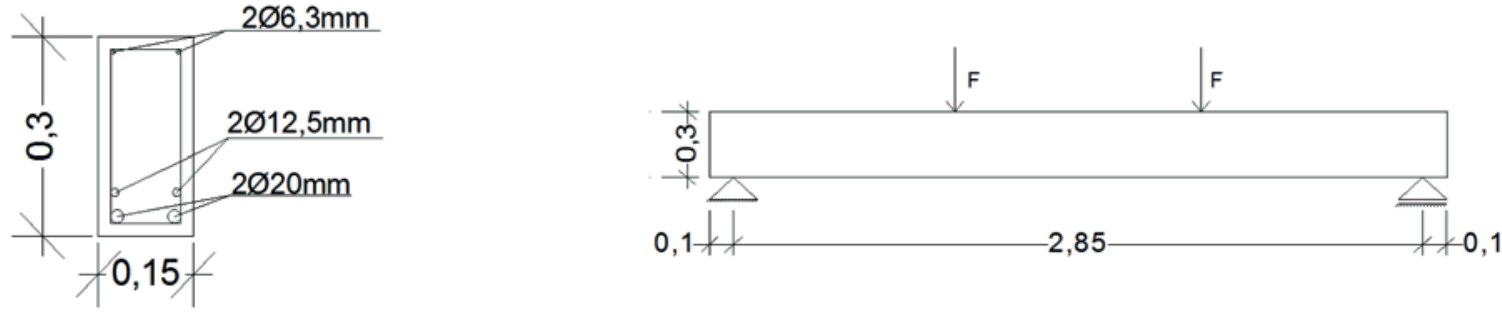


\section{Table 3 - Parameter values of the proposed damage model - RC Beam}

\begin{tabular}{cc} 
Tension & Compression \\
$\mathrm{Y}_{01}=6.0 \times 10^{-5} \mathrm{MPa}$ & $\mathrm{Y}_{02}=1.0 \times 10^{-3} \mathrm{MPa}$ \\
$\mathrm{A}_{1}=0.3$ & $\mathrm{~A}_{2}=1.5$ \\
$\mathrm{~B}_{1}=195 \mathrm{MPa}^{-1}$ & $\mathrm{~B}_{2}=10.2 \mathrm{MPa}^{-1}$ \\
$\beta_{1}=5 \times 10^{-5} \mathrm{MPa}$ & $\beta_{2}=3 \times 10^{-4} \mathrm{MPa}$ \\
\hline
\end{tabular}

simplified 1D version has been used. The 1D analysis has shown an efficient and practical employment in the simulation of the damage processes in framed structures submitted to inverse loading, without numerical problems and low computational cost. Besides, the parametric identification is simple. In this case, the damage model could be used in estimative analyses of structures in practical situations, such as: numerical simulation of displacement in cracking concrete beams submitted to service loads, estimative of ultimate load capacity of frames and beams and collapse configuration of reinforced concrete frames.

The obtained results encourage us to proceed in the improvement of the model to deal with more complex phenomena in future works, e. g., blocking and dissipative sliding of closed microcracks lips, non-local version of the model and a more efficient parametric identification of $\beta_{1}$ and $\beta_{2}$, among others. Besides, the damage model can be improved with the consideration of the shear contribution of the concrete for the mechanical behaviour of framed structures even using 1D modelling.

\section{Ackowledgements}

The authors wish to thank to CNPq (National Council for Scientific and Technological Development) and CAPES (Foundation, Ministry of Education of Brazil) for the financial support.

\section{References}

[01] Curnier, A. He, Q. and Zysset, P. (1995), "Conewise linear elastic materials", J Elasticity, 37, 1-38.

[02] Pituba, J. J. C. (2006), "On the formulation of damage constitutive models for bimodular anistropic media". In: C. A. M. Soares (ed) European conference on computational mechanics, 3rd edn. Springer, pp 371. DOI: 10.1007/1-40205370-3_371.

[03] Pituba, J. J. C. (2010), "Validation of a damage model for the non linear analysis of reinforced concrete structures", Acta Sci-Technol. 3, 251-259. DOI: 10.4025/actascitechnol. v32i3.7009.

[04] Pituba, J. J. C. and Fernandes, G. R. (2011), "An anisotropic damage model for concrete", J Eng Mech-ASCE. 137, 610624. DOI: 10.1061/(ASCE)EM.1943-7889.0000260.

[05] Pituba, J. J. C. and Lacerda, M. M. S. (2012), "Simplified damage models applied in the numerical analysis of $r$ einforced concrete structures", Rev IBRACON Estrut Mater., 1, 26-37.

[06] Pituba, J. J. C. , Delalibera, R. G. and Rodrigues, F. S.
(2012), "Numerical and statistical analysis about displacements in reinforced concrete beams using damage mechanics", Comput Concrete. 10, 307-330.

[07] Comi, C. (2001), "A non-local model with tension and compression damage mechanisms", Eur J Mech A-Solid. 20, $1-22$

[08] Desmorat, R. (2000), "Strain localization and unilateral conditions for anisotropic induced damage model", In: Proc. Continuous Damage and Fracture, Cachan, 71-79.

[09] Carol, I. and Willam, K. (1996), "Spurious Energy dissipation/ generation in stiffness recovery models for elastic degradation and damage", Int J Solids Struct., 33, 2939-257.

[10] Welemane, H. and Cormery, F. (2002), "Some remarks on the damage unilateral effect modeling for microcracked materials", Int J Damage Mech., 11, 65-86.

[11] Bielski, J., Skrzypek, J. J. and Kuna-Cisal, H. (2006), "Implementation of a model of coupled elastic-plastic unilateral damage material to finite element code", Int J Damage Mech., 15, 5-39.

[12] Liu, Y., Teng, S. and Soh, C. (2008), "Three-Dimensional Damage Model for Concrete. II: Verification”, J Eng MechASCE., 132, 82-89.

[13] Araújo, F. A. and Proença, S. P. B. (2008), "Application of a lumped dissipation model to reinforced concrete structures with the consideration of residual strains and cycles of hysteresis", J Mech Mater Struct., 3, 1011-1031.

[14] Zhu, Q., Kondo, D., Shao, J. and Pensee, V. (2008), "Micromechanical modelling of anisotropic damage in brittle rocks and application", Int J Rock Mech Min., 45, 467-477.

[15] Zhu, Q., Kondo, D. and Shao, J. (2009), "Homogenizationbased analysis of anisotropic damage in brittle materials with unilateral effect and interactions between microcracks", Int $J$ Numer Anal Met., 33, 749-772.

[16] Pichler, B. and Dormieux, L. (2009), "Instability during cohesive zone growth", Eng Fract Mech., 76, 1729-1749.

[17] Skarzynski, L. and Tejchman, J. (2012), "Determination of representative volume element in concrete under tensile deformation", Comput Concrete., 9, 35-50.

[18] Pituba, J. J. C. and Souza Neto, E. A. (2012), "Characterization of macroscopic mechanical behavior of concrete with mesoscopic scale finite element analysis", In: J. Eberhardsteiner, H. J. Böhm, F. G. Rammerstorfer (eds), European Congress on Computational Methods in Applied Sciences and Engineering, Vienna University of Technology, Viena, ISBN: 978-3-9502481-9-7.

[19] Matallah, M. and La Borderie, C.I (2009), "Inelasticity-damage-based model for numerical modeling of concrete cracking", Eng Fract Mech., 76, 1087-1108.

[20] La Borderie, C. (1991), "Phenomenes unilateraux dans un materiau endommageable: modelisation et application a l'analyse de structures en beton", Doctoral Dissertation, University of Paris 6, France.

[21] Vecchio, F. J. and Emara, M. B. (1992), "Shear deformations in reinforced concrete frames", ACl Struct J., 89, 46-56.

[22] Nogueira, C. G., Venturini, W. S. and Coda, H. B. (2013), "Material and geometric nonlinear analysis of reinforced concrete frame structures considering the influence of shear strength complementary mechanisms", Latin American Journal of Solids and Structures, 10, 953-980. 